\title{
POSITIVITY IN $T$-EQUIVARIANT $K$-THEORY OF FLAG VARIETIES ASSOCIATED TO KAC-MOODY GROUPS II
}

\author{
SETH BALDWIN AND SHRAWAN KUMAR
}

\begin{abstract}
We prove sign-alternation of the structure constants in the basis of the structure sheaves of opposite Schubert varieties in the torus-equivariant Grothendieck group of coherent sheaves on the flag varieties $G / P$ associated to an arbitrary symmetrizable Kac-Moody group $G$, where $P$ is any parabolic subgroup. This generalizes the work of Anderson-Griffeth-Miller from the finite case to the general Kac-Moody case, and affirmatively answers a conjecture of Lam-Schilling-Shimozono regarding the signs of the structure constants in the case of the affine Grassmannian.
\end{abstract}

\section{INTRODUCTION}

Let $G$ be any symmetrizable Kac-Moody group over $\mathbb{C}$ completed along the negative roots and $G^{\text {min }} \subset G$ the minimal Kac-Moody group as in [Ku2, $\left.§ 7.4\right]$. Let $B$ be the standard (positive) Borel subgroup, $B^{-}$the standard negative Borel subgroup, $H=B \cap B^{-}$the standard maximal torus, and $W$ the Weyl group [Ku2, Chapter 6$]$. Let $\bar{X}=G / B$ be the 'thick' flag variety (introduced by Kashiwara) which contains the standard flag variety $X=G^{\mathrm{min}} / B$. Let $T$ be the adjoint torus, i.e., $T:=H / Z\left(G^{\mathrm{min}}\right)$, where $Z\left(G^{\mathrm{min}}\right)$ denotes the center of $G^{\mathrm{min}}$, and let $R(T)$ denote the representation ring of $T$. For any $w \in W$, we have the Schubert cell $C_{w}:=B w B / B \subset X$, the Schubert variety $X_{w}:=\overline{C_{w}} \subset X$, the opposite Schubert cell $C^{w}:=B^{-} w B / B \subset \bar{X}$, and the opposite Schubert variety $X^{w}:=\overline{C^{w}} \subset \bar{X}$. When $G$ is a (finite dimensional) semisimple group, it is referred to as the finite case.

Let $K_{T}^{0}(\bar{X})$ denote the Grothendieck group of $T$-equivariant coherent sheaves on $\bar{X}$. Then, $\left\{\left[\mathcal{O}_{X^{w}}\right]\right\}_{w \in W}$ forms an $R(T)$-'basis' of $K_{T}^{0}(\bar{X})$ (where infinite sums are allowed), i.e., $K_{T}^{0}(\bar{X})=\prod_{w \in W} R(T)\left[\mathcal{O}_{X^{w}}\right]$. We express the product in $K_{T}^{0}(\bar{X})$ by:

$$
\left[\mathcal{O}_{X^{u}}\right] \cdot\left[\mathcal{O}_{X^{v}}\right]=\sum_{w \in W} d_{u, v}^{w}\left[\mathcal{O}_{X^{w}}\right], \text { for unique } d_{u, v}^{w} \in R(T) .
$$

The following result is our main theorem (cf. Theorem 5.3). This was conjectured first by Griffeth-Ram GR] in the finite case (2004), proven in the finite case by Anderson-Griffeth-Miller AGM (2011), and then conjectured in the general KacMoody case by Kumar [Ku1] (2012).

Theorem 1.1. For any $u, v, w \in W$,

$$
(-1)^{\ell(w)+\ell(u)+\ell(v)} d_{u, v}^{w} \in \mathbb{Z}_{\geq 0}\left[\left(e^{-\alpha_{1}}-1\right), \ldots,\left(e^{-\alpha_{r}}-1\right)\right],
$$

where $\left\{\alpha_{1} \ldots, \alpha_{r}\right\}$ are the simple roots.

Received by the editors December 4, 2016.

2010 Mathematics Subject Classification. Primary 19L47; Secondary 14M15. 
Let $P$ be any standard parabolic subgroup of $G$ of finite type (cf. [Ku2, Definition 6.1.18]). (Recall that a parabolic subgroup is said to be of finite type if its Levi subgroup is finite dimensional.) We may express, in $K_{T}^{0}(G / P)$,

$$
\left[\mathcal{O}_{X_{P}^{u}}\right] \cdot\left[\mathcal{O}_{X_{P}^{v}}\right]=\sum_{w \in W^{P}} d_{u, v}^{w}(P)\left[\mathcal{O}_{X_{P}^{w}}\right], \text { for unique } d_{u, v}^{w}(P) \in R(T),
$$

where $W^{P}$ is the set of minimal length representatives of $W / W_{P}, W_{P}$ is the Weyl group of $P$, and $X_{P}^{w}:=\overline{B^{-} w P / P} \subset G / P$ is the opposite Schubert variety.

Let $\pi: G / B \rightarrow G / P$ be the standard (T-equivariant) projection. Then, $\pi$ is a locally trivial fibration (with fiber the smooth projective variety $P / B$ ) and hence flat (cf. [Ku2, Chapter 7]). Thus, we have

$$
\pi^{*}\left[\mathcal{O}_{X_{P}^{w}}\right]=\left[\mathcal{O}_{\pi^{-1}\left(X_{P}^{w}\right)}\right]=\left[\mathcal{O}_{X^{w}}\right] .
$$

Since $\pi^{*}: K_{T}^{0}(G / P) \rightarrow K_{T}^{0}(G / B)$ is a ring homomorphism, we have $d_{u, v}^{w}=d_{u, v}^{w}(P)$ for any $u, v, w \in W^{P}$ and hence Theorem 1.1 immediately generalizes from the case of $G / B$ to the case of $G / P$, and we obtain:

Theorem 1.2. For any standard parabolic subgroup $P$ of $G$ of finite type, and any $u, v, w \in W^{P}$,

$$
(-1)^{\ell(w)+\ell(u)+\ell(v)} d_{u, v}^{w}(P) \in \mathbb{Z}_{\geq 0}\left[\left(e^{-\alpha_{1}}-1\right), \ldots,\left(e^{-\alpha_{r}}-1\right)\right] .
$$

Theorems 1.1 and 1.2 also apply to ordinary (non-equivariant) $K$-theory. Let $K^{0}(\bar{X})$ denote the Grothendieck group of coherent sheaves on $\bar{X}$. Then, we have $K^{0}(\bar{X})=\prod_{w \in W} \mathbb{Z}\left[\mathcal{O}_{X^{w}}\right]$. Further, the map

$$
\mathbb{Z} \otimes_{R(T)} K_{T}^{0}(\bar{X}) \rightarrow K^{0}(\bar{X}), 1 \otimes\left[\mathcal{O}_{X} w\right] \mapsto\left[\mathcal{O}_{X} w\right],
$$

is an isomorphism, where we view $\mathbb{Z}$ as an $R(T)$-module via evaluation at 1 . Similar results apply to $G / P$.

Write, in $K^{0}(G / P)$, for $u, v \in W^{P}$,

$$
\left[\mathcal{O}_{X_{P}^{u}}\right] \cdot\left[\mathcal{O}_{X_{P}^{v}}\right]=\sum_{w \in W^{P}} a_{u, v}^{w}(P)\left[\mathcal{O}_{X_{P}^{w}}\right], \text { for unique } a_{u, v}^{w}(P) \in \mathbb{Z} .
$$

Then, by the above, along with Theorem 1.2 we have:

Theorem 1.3. For any standard parabolic subgroup $P$ of $G$ of finite type, and any $u, v, w \in W^{P}$,

$$
(-1)^{\ell(w)+\ell(u)+\ell(v)} a_{u, v}^{w}(P) \in \mathbb{Z}_{\geq 0} .
$$

The following conjecture of Lam-Schilling-Shimozono [LSS, Conjectures 7.20 (ii) and 7.21 (iii)] is a special case of Theorem 1.3

Let $G=\widehat{S L_{N}}$ be the affine Kac-Moody group associated to $S L_{N}$, and let $P$ be its standard maximal parabolic subgroup. Let $\overline{\mathcal{X}}=G / P$ be the corresponding infinite Grassmannian. Then, $K^{0}(\overline{\mathcal{X}})$ has the structure sheaf 'basis' $\left\{\left[\mathcal{O}_{X^{u}}\right]\right\}_{u \in W^{P}}$ over $\mathbb{Z}$, where $W$ is the (affine) Weyl group of $G$ and $W^{P}$ is the set of minimal coset representatives in $W / W_{o}$ ( $W_{o}$ being the finite Weyl group of $\left.S L_{N}\right)$. Write, for any $u, v \in W^{P}$,

$$
\left[\mathcal{O}_{X^{u}}\right] \cdot\left[\mathcal{O}_{X^{v}}\right]=\sum_{w \in W^{P}} b_{u, v}^{w}\left[\mathcal{O}_{X^{w}}\right], \text { for unique integers } b_{u, v}^{w} .
$$


Then, the following is a special case of Theorem 1.3 .

Corollary 1.4 (Conjectured by Lam-Schilling-Shimozono).

$$
(-1)^{\ell(u)+\ell(v)+\ell(w)} b_{u, v}^{w} \in \mathbb{Z}_{\geq 0} .
$$

The proof of Theorem 1.1 follows closely the work of Anderson-Griffeth-Miller [AGM] and Kumar [Ku1; though several technical details had to be carefully addressed, e.g., Proposition 7.7 proving the rational singularities of $\mathcal{Z}$. Letting $K_{0}^{T}(X)$ denote the Grothendieck group of finitely supported $T$-equivariant coherent sheaves on $X$, there is a natural pairing

$$
\langle,\rangle: K_{T}^{0}(\bar{X}) \otimes K_{0}^{T}(X) \rightarrow R(T)
$$

coming from the $T$-equivariant Euler-Poincaré characteristic (cf. (10)). Under this pairing, the bases $\left\{\left[\mathcal{O}_{X^{u}}\right]\right\}$ of $K_{T}^{0}(\bar{X})$ and $\left\{\left[\xi_{w}\right]\right\}$ of $K_{0}^{T}(X)$ are dual (cf. Proposition 3.10), where $\xi_{w}:=\mathcal{O}_{X_{w}}\left(-\partial X_{w}\right)$ and $\partial X_{w}:=X_{w} \backslash C_{w}$. (By Lemma 3.5, $\xi_{w}$ is indeed a basis of $K_{0}^{T}(X)$.)

Then, realizing the product structure constants in $K_{T}^{0}(\bar{X})$ as the coproduct structure constants of the dual basis in $K_{0}^{T}(X)$ (cf. Lemma 4.2) allows the use of the above pairing and duality to express the structure constants in terms of certain cohomology groups. Following [AGM], we introduce the 'mixing space' $X_{\mathbb{P}}$, which is a bundle over a product of projective spaces $\mathbb{P}$ with fiber $X$. This allows for the reduction from $T$-equivariant $K$-theory to non-equivariant $K$-theory. Then, we introduce the 'mixing group' $\Gamma$ (cf. Definition 4.11) whose action is sufficient to ensure a certain transverality needed to prove our main result.

Theorem 5.1 is our main technical result. Part a) allows the structure constants to be realized as the Euler characteristic of a certain sheaf, while part b) shows that this sheaf has cohomology which is zero in all but a single term of known degree, immediately resulting in a determination of the sign of the Euler characteristic and hence the structure constants.

Part a) of Theorem 5.1 is proved in Section 6. The proof relies on some local $\mathscr{T}$ or vanishing results which were proven in [Ku1, as well as a reduction to finite dimensional schemes, where a transversality result due to Sierra is crucially used (cf. Theorem 6.1).

The proof of the more difficult part b) of Theorem 5.1 is the content of Sections 7 through 9. In Section 7 we introduce the crucial scheme $\mathcal{Z}$ which comes with a projection to the mixing group $\Gamma$. We also introduce a desingularization $\widetilde{\mathcal{Z}}$ of $\mathcal{Z}$ and prove that $\mathcal{Z}$ is irreducible, normal and has rational singularities (cf. Propositions 7.5 and (7.7). We further introduce the divisor $\partial \mathcal{Z} \subset \mathcal{Z}$ which is shown to be CohenMacaulay (cf. Proposition 7.8). It is on the fibers of the projection $\pi: \mathcal{Z} \rightarrow \Gamma$ that the computation of the cohomology of the previously mentioned sheaf occurs.

In Section 8 the rational singularities of $\mathcal{Z}$ are used to apply a relative version of the Kawamata-Viehweg vanishing theorem (cf. Theorem 8.1) to show that $R^{i} \pi_{*} \omega_{\mathcal{Z}}(\partial \mathcal{Z})=0$ for all $i>0$ (cf. Corollary 8.8). Finally, in Section 9, this vanishing of the higher direct images along with the semicontinuity theorem is used to prove vanishing of the relevant cohomology along the fibers of $\pi: \mathcal{Z} \rightarrow \Gamma$ (cf. Theorem 9.2) and thus conclude the proof of part b) of Theorem 5.1 . 


\section{Notation}

We work over the field $\mathbb{C}$ of complex numbers. By a variety we mean an algebraic variety over $\mathbb{C}$ which is reduced, but not necessarily irreducible.

Let $G$ be any symmetrizable Kac-Moody group over $\mathbb{C}$ completed along the negative roots (as opposed to completed along the positive roots as in $\mathrm{Ku} 2$, Chapter 6]) and $G^{\text {min }} \subset G$ the minimal Kac-Moody group as in [Ku2, §7.4]. Let $B$ be the standard (positive) Borel subgroup, $B^{-}$the standard negative Borel subgroup, $H=B \cap B^{-}$the standard maximal torus, and $W$ the Weyl group [Ku2, Chapter 6]. Let

$$
\bar{X}=G / B
$$

be the 'thick' flag variety which contains the standard flag variety $X=G^{\text {min }} / B$.

If $G$ is not of finite type, $\bar{X}$ is an infinite dimensional non-quasi-compact scheme $[\mathrm{K}, \S 4]$ and $X$ is an ind-projective variety $\mathrm{Ku} 2, \S 7.1]$. The group $G^{\mathrm{min}}$ and, in particular, the maximal torus $H$ acts on $\bar{X}$ and $X$.

Let $T$ be the adjoint torus, i.e., $T:=H / Z\left(G^{\text {min }}\right)$, where $Z\left(G^{\text {min }}\right)$ denotes the center of $G^{\mathrm{min}}$. (Recall that by [Ku2, Lemma 6.2.9(c)], $Z\left(G^{\mathrm{min}}\right)=\{h \in H$ : $e^{\alpha_{i}}(h)=1$ for all the simple roots $\left.\alpha_{i}\right\}$.) Then, the action of $H$ on $\bar{X}$ (and on $X$ ) descends to an action of $T$.

For any $w \in W$, we have the Schubert cell

$$
C_{w}:=B w B / B \subset X,
$$

the Schubert variety

$$
X_{w}:=\overline{C_{w}}=\bigsqcup_{w^{\prime} \leq w} C_{w^{\prime}} \subset X,
$$

the opposite Schubert cell

$$
C^{w}:=B^{-} w B / B \subset \bar{X}
$$

and the opposite Schubert variety

$$
X^{w}:=\overline{C^{w}}=\bigsqcup_{w^{\prime} \geq w} C^{w^{\prime}} \subset \bar{X}
$$

all endowed with the reduced subscheme structures. Then, $X_{w}$ is a (finite dimensional) irreducible projective subvariety of $X$ and $X^{w}$ is a finite codimensional irreducible subscheme of $\bar{X}[\mathrm{Ku} 2, \S 7.1]$ and $[\mathrm{K}, \S 4]$. We denote by $Z_{w}$ the Bott-Samelson-Demazure-Hansen $(\mathrm{BSDH})$ variety as in [Ku2, §7.1.3], which is a $B$-equivariant desingularization of $X_{w}\left[\mathrm{Ku} 2\right.$, Proposition 7.1.15]. Further, $X_{w}$ is normal, Cohen-Macaulay (CM for short) and has rational singularities Ku2, Theorem 8.2.2].

We also define the boundary of the Schubert variety by

$$
\partial X_{w}:=X_{w} \backslash C_{w}
$$

with the reduced subscheme structure. Then, $\partial X_{w}$ is pure of codimension 1 in $X_{w}$ and is CM (See the proof of Proposition 7.8).

For any $u \leq w$, we have the Richardson variety

$$
X_{w}^{u}:=X^{u} \cap X_{w} \subset X
$$


endowed with the reduced subvariety structure. By [Ku1, Proposition 6.6], $X_{w}^{u}$ is irreducible, normal and CM. We denote by $Z_{w}^{u}$ the $T$-equivariant desingularization of $X_{w}^{u}$ as in [Ku1, Theorem 6.8]. By [KuS, Theorem 3.1], $X_{w}^{u}$ has rational singularities (in fact it has Kawamata log terminal singularities).

We denote the representation ring of $T$ by $R(T)$. Let $\left\{\alpha_{1}, \ldots, \alpha_{r}\right\} \subset \mathfrak{h}^{*}$ be the set of simple roots, $\left\{\alpha_{1}^{\vee}, \ldots, \alpha_{r}^{\vee}\right\} \subset \mathfrak{h}$ the set of simple coroots, and $\left\{s_{1}, \ldots, s_{r}\right\}$ the corresponding set of simple reflections, where $\mathfrak{h}:=\operatorname{Lie}(H)$. Let $\rho \in \mathfrak{h}^{*}$ be any integral weight satisfying

$$
\rho\left(\alpha_{i}^{\vee}\right)=1, \text { for all } 1 \leq i \leq r .
$$

When $G$ is a finite dimensional semisimple group, $\rho$ is unique, but for a general Kac-Moody group $G$, it may not be unique.

For any integral weight $\lambda$ let $\mathbb{C}_{\lambda}$ denote the one-dimensional representation of $H$ on $\mathbb{C}$ given by $h \cdot v=\lambda(h) v$ for $h \in H, v \in \mathbb{C}$. By extending this action to $B$ we may define, for any integral weight $\lambda$, the $G$-equivariant line bundle $\mathcal{L}(\lambda)$ on $\bar{X}$ by

$$
\mathcal{L}(\lambda):=G \times{ }^{B} \mathbb{C}_{-\lambda},
$$

where, for any representation $V$ of $B, G \times{ }^{B} V:=(G \times V) / B$ and $B$ acts on $G \times V$ via $(g, v) \cdot b=\left(g b, b^{-1} v\right)$ for $g \in G, v \in V, b \in B$. Then, $G \times{ }^{B} V$ is the total space of a $G$-equivariant vector bundle over $X$, with projection given by $(g, v) B \mapsto g B$. We also define the bundle

$$
e^{\lambda}:=\bar{X} \times \mathbb{C}_{\lambda},
$$

which while trivial when viewed as a non-equivariant line bundle, is equivariantly non-trivial with the diagonal action of $H$.

\section{The GRothendieCK GROUP}

For a quasi-compact scheme $Y$, an $\mathcal{O}_{Y}$-module is called coherent if it is finitely presented as an $\mathcal{O}_{Y}$-module and any $\mathcal{O}_{Y}$-submodule of finite type admits a finite presentation.

A subset $S \subset W$ is called an ideal if for all $x \in S$ and $y \leq x$ we have $y \in S$. We say that an $\mathcal{O}_{\bar{X}}$-module $\mathcal{S}$ is coherent if $\left.\mathcal{S}\right|_{V^{S}}$ is a coherent $\mathcal{O}_{V^{S}}$-module for every finite ideal $S \subset W$, where $V^{S}$ is the quasi-compact open subset of $\bar{X}$ defined by

$$
V^{S}:=\bigcup_{w \in S} w U^{-} B / B, \text { where } U^{-}:=\left[B^{-}, B^{-}\right] .
$$

Let $K_{T}^{0}(\bar{X})$ denote the Grothendieck group of $T$-equivariant coherent $\mathcal{O}_{\bar{X}}$-modules. Since the coherence condition on $\mathcal{S}$ is imposed only for $\left.\mathcal{S}\right|_{V^{S}}$ for finite ideals $S \subset W$, $K_{T}^{0}(\bar{X})$ can be thought of as the inverse limit of $K_{T}^{0}\left(V^{S}\right)$ as $S$ varies over all finite ideals of $W$ (cf. [KS, §2]).

We define

$$
K_{0}^{T}(X):=\operatorname{Limit}_{n \rightarrow \infty} K_{0}^{T}\left(X_{n}\right),
$$

where $\left\{X_{n}\right\}_{n \geq 1}$ is the filtration of $X$ giving the ind-projective variety structure and $K_{0}^{T}\left(X_{n}\right)$ is the Grothendieck group of $T$-equivariant coherent sheaves on $X_{n}$.

For any $u \in W, \mathcal{O}_{X^{u}}$ is a coherent $\mathcal{O}_{\bar{X}}$-module by [KS, §2]. From [KS, comment after Remark 2.4] we have:

Lemma 3.1. $\left\{\left[\mathcal{O}_{X^{u}}\right]\right\}$ forms a basis of $K_{T}^{0}(\bar{X})$ as an $R(T)$-module (where we allow arbitrary infinite sums).

Lemma 3.2. $\left\{\left[\mathcal{O}_{X_{w}}\right]\right\}$ forms a basis of $K_{0}^{T}(X)$ as an $R(T)$-module. 
Proof. This follows from [CG, §5.2.14 and Theorem 5.4.17].

The following lemma is due to Kashiwara-Shimozono [KS, Lemma 8.1].

Lemma 3.3. Any $T$-equivariant coherent sheaf $\mathcal{S}$ on $V^{u}$ admits a free resolution in $\operatorname{Coh}_{T}\left(\mathcal{O}_{V^{u}}\right)$ :

$$
0 \rightarrow S_{n} \otimes \mathcal{O}_{V^{u}} \rightarrow \cdots \rightarrow S_{1} \otimes \mathcal{O}_{V^{u}} \rightarrow S_{0} \otimes \mathcal{O}_{V^{u}} \rightarrow \mathcal{S} \rightarrow 0,
$$

where $S_{k}$ are finite dimensional T-modules, $V^{u}:=u U^{-} B / B \subset \bar{X}$, and $\operatorname{Coh}_{T}\left(\mathcal{O}_{V^{u}}\right)$ denotes the abelian category of $T$-equivariant coherent $\mathcal{O}_{V^{u}}$-modules.

We define a pairing

$$
\begin{gathered}
\langle,\rangle: K_{T}^{0}(\bar{X}) \otimes K_{0}^{T}(X) \rightarrow R(T), \\
\langle[\mathcal{S}],[\mathcal{F}]\rangle=\sum_{i}(-1)^{i} \chi_{T}\left(X_{n}, \mathscr{T}_{\text {or }}^{\mathcal{O}_{i}} \bar{x}(\mathcal{S}, \mathcal{F})\right),
\end{gathered}
$$

where $\mathcal{S}$ is a $T$-equivariant coherent sheaf on $\bar{X}$ and $\mathcal{F}$ is a $T$-equivariant coherent sheaf on $X$ supported on $X_{n}$ for some $n$, where $\chi_{T}$ represents the $T$-equivariant Euler-Poincaré characteristic. By [Ku1, Lemma 3.5] this is well defined.

Definition 3.4. We define $\xi_{w}$ to be the ideal sheaf of $\partial X_{w}$ in $X_{w}$, where $\partial X_{w}$ is given the reduced subscheme structure:

$$
\xi_{w}:=\mathcal{O}_{X_{w}}\left(-\partial X_{w}\right)
$$

Lemma 3.5. $\left\{\left[\xi_{w}\right]\right\}$ forms a basis of $K_{0}^{T}(X)$ as an $R(T)$-module.

Proof. This follows since $\left[\xi_{w}\right]=\left[\mathcal{O}_{X_{w}}\right]+\sum_{w^{\prime}<w} r_{w^{\prime}}\left[\mathcal{O}_{X_{w^{\prime}}}\right]$, for some $r_{w^{\prime}} \in R(T)$ and the fact that $\left[\mathcal{O}_{X_{w}}\right]$ is a basis of $K_{0}^{T}(X)$ (cf. Lemma [3.2).

Proposition 3.6. $\omega_{X_{w}}=e^{-\rho} \mathscr{L}(-\rho) \xi_{w}$, where $\omega_{X_{w}}$ is the dualizing sheaf of $X_{w}$.

Proof. This follows from [GK, Proposition 2.2] since the same proof works for general Kac-Moody groups.

From [Ku1, Lemma 5.5] we have the following result:

Lemma 3.7. For any $u, w \in W$, we have

$$
\mathscr{T}_{\text {or }} \mathcal{O}_{\bar{X}}\left(\mathcal{O}_{X^{u}}, \mathcal{O}_{X_{w}}\right)=0, \forall i>0
$$

We now prove:

Lemma 3.8. For any $u \in W$ and any finite union of Schubert varieties $Y=$ $\bigcup_{i=1}^{\ell} X_{w_{i}}$ we have

$$
\mathscr{T}_{\text {or }}^{\mathcal{O}_{i}}{ }_{\bar{X}}\left(\mathcal{O}_{X^{u}}, \mathcal{O}_{Y}\right)=0, \forall i>0 .
$$

In particular, for any $u, w \in W$ we have

$$
\mathscr{T}_{\text {or }} \boldsymbol{\mathcal { O }}_{\bar{X}}\left(\mathcal{O}_{X^{u}}, \mathcal{O}_{\partial X_{w}}\right)=0, \forall i>0 .
$$

Proof. We proceed by double induction on the dimension of $Y$ (i.e., the largest dimension of the irreducible components of $Y$ ) and the number of irreducible components of $Y$. If $\operatorname{dim} Y=0$, then $Y=X_{e}$, and so the result follows from Lemma 3.7. Now, suppose that $\operatorname{dim} Y=d$ and $Y$ has $k$ irreducible components. If $k=1$, then the result follows from Lemma 3.7. so we may assume that $k \geq 2$. Let $Y_{1}=X_{w}$ be an irreducible component of $Y$ and let $Y_{2}$ be the union of all the other irreducible components. 
By [ $\mathrm{KuS}$, Proposition 5.3 and its proof $X$ is Frobenius split compatibly splitting its Schubert varieties $X_{u}$ and also Richardson varieties $X_{u}^{v}$.

(*) Thus, any finite intersection

$$
X_{u_{1}} \cap \ldots \cap X_{u_{n}} \cap X^{v_{1}} \cap \ldots \cap X^{v_{m}} \text { is reduced for any } n \geq 1 \text {. }
$$

(In the proof here we have only used $X_{u_{1}} \cap \ldots \cap X_{u_{n}}$ to be reduced, but the more general assertion here will be used later in the paper.)

The short exact sequence of sheaves

$$
0 \rightarrow \mathcal{O}_{Y} \rightarrow \mathcal{O}_{Y_{1}} \oplus \mathcal{O}_{Y_{2}} \rightarrow \mathcal{O}_{Y_{1} \cap Y_{2}} \rightarrow 0
$$

yields the long exact sequence

$$
\begin{aligned}
\ldots \rightarrow \mathscr{T} \operatorname{or}_{i+1}^{\mathcal{O}_{\bar{X}}}\left(\mathcal{O}_{X^{u}}, \mathcal{O}_{Y_{1} \cap Y_{2}}\right) & \rightarrow \mathscr{T} r_{i}^{\mathcal{O}_{\bar{X}}}\left(\mathcal{O}_{X^{u}}, \mathcal{O}_{Y}\right) \\
& \rightarrow \mathscr{T}_{o r}^{\mathcal{O}_{\bar{X}}^{\bar{X}}}\left(\mathcal{O}_{X^{u}}, \mathcal{O}_{Y_{1}} \oplus \mathcal{O}_{Y_{2}}\right) \rightarrow \ldots
\end{aligned}
$$

Now, since $Y_{2}$ has less than $k$ irreducible components, induction on the number of irreducible components gives

$$
\mathscr{T}_{\text {or }}^{\mathcal{O}_{\bar{X}}}\left(\mathcal{O}_{X^{u}}, \mathcal{O}_{Y_{1}} \oplus \mathcal{O}_{Y_{2}}\right)=0, \forall i>0 .
$$

Since $Y_{1} \cap Y_{2}$ is reduced and of dimension less than $d$, induction on the dimension gives

$$
\mathscr{T}_{\operatorname{or}}^{\mathcal{O}_{\bar{X}}}\left(\mathcal{O}_{X^{u}}, \mathcal{O}_{Y_{1} \cap Y_{2}}\right)=0, \forall i>0 .
$$

Together, these imply the lemma.

Lemma 3.9. For any $u, w \in W$, we have

$$
\mathscr{T}_{\text {or }}^{\mathcal{O}_{\bar{X}}}\left(\mathcal{O}_{X^{u}}, \xi_{w}\right)=0, \forall i>0 .
$$

Proof. Applying Lemmas 3.7 and 3.8 the desired result follows from the long exact sequence for $\mathscr{T}$ or.

Proposition 3.10. For any $u, w \in W$ we have

$$
\left\langle\left[\mathcal{O}_{X^{u}}\right],\left[\xi_{w}\right]\right\rangle=\delta_{u, w} .
$$

Proof. By definition

$$
\left\langle\left[\mathcal{O}_{X^{u}}\right],\left[\xi_{w}\right]\right\rangle=\sum_{i}(-1)^{i} \chi_{T}\left(X_{n}, \mathscr{T}_{\operatorname{or}_{i}}^{\mathcal{O}_{\bar{X}}}\left(\mathcal{O}_{X^{u}}, \xi_{w}\right)\right),
$$

where $n$ is taken such that $n \geq \ell(w)$. Thus, by Lemma 3.9, we have

$$
\left\langle\left[\mathcal{O}_{X^{u}}\right],\left[\xi_{w}\right]\right\rangle=\chi_{T}\left(X_{n}, \mathcal{O}_{X^{u}} \otimes_{\mathcal{O}_{\bar{X}}} \xi_{w}\right) .
$$

By Lemma 3.8 we have the sheaf exact sequence

$$
0 \rightarrow \mathcal{O}_{X^{u}} \otimes_{\mathcal{O}_{\bar{X}}} \xi_{w} \rightarrow \mathcal{O}_{X^{u}} \otimes_{\mathcal{O}_{\bar{X}}} \mathcal{O}_{X_{w}} \rightarrow \mathcal{O}_{X^{u}} \otimes_{\mathcal{O}_{\bar{X}}} \mathcal{O}_{\partial X_{w}} \rightarrow 0
$$

Observe that by (荬)

$$
\mathcal{O}_{X^{u}} \otimes_{\mathcal{O}_{\bar{X}}} \mathcal{O}_{X_{w}}=\mathcal{O}_{X^{u} \cap X_{w}}
$$

and similarly for $\mathcal{O}_{X^{u}} \otimes_{\mathcal{O}_{\bar{X}}} \mathcal{O}_{\partial X_{w}}$. Thus,

$$
\chi_{T}\left(X_{n}, \mathcal{O}_{X^{u}} \otimes_{\mathcal{O}_{\bar{X}}} \xi_{w}\right)=\chi_{T}\left(X_{n}, \mathcal{O}_{X^{u} \cap X_{w}}\right)-\chi_{T}\left(X_{n}, \mathcal{O}_{X^{u} \cap \partial X_{w}}\right) .
$$


When non-empty, $X^{u} \cap X_{w}$ is irreducible by [Ku1, Proposition 6.6], and thus $X^{u} \cap \partial X_{w}=\bigcup_{u<w^{\prime}<w} X^{u} \cap X_{w^{\prime}}$ is a connected projective variety when non-empty, since $u \in X^{u} \cap \bar{X}_{w^{\prime}}$ for all $u \leq w^{\prime}<w$.

If $u \not \leq w$ we have $X^{u} \cap X_{w}=\emptyset$, so assume $u \leq w$. In this case $X^{u} \cap X_{w} \neq \emptyset$. Now, if $u=w$, then $X^{u} \cap \partial X_{w}=\emptyset$, while if $u<w$, then $X^{u} \cap \partial X_{w} \neq \emptyset$. By KuS, Corollary 3.2],

$$
H^{i}\left(X_{n}, \mathcal{O}_{X^{u} \cap X_{w}}\right)=0, \forall i>0
$$

Using an inductive argument similar to Lemma 3.8 we obtain

$$
H^{i}\left(X_{n}, \mathcal{O}_{X^{u} \cap Y}\right)=0, \forall i>0
$$

where $Y$ is any finite union of Schubert varieties. Taking $Y=X^{u} \cap \partial X_{w}$ and combining the above implies the proposition (here we use that, when non-empty, $X^{u} \cap Y$ is connected).

\section{The MiXing SPACE AND MIXING GRoup}

In this section we realize the product structure constants of $\left\{\left[\mathcal{O}_{X^{u}}\right]\right\}$ in $K_{T}^{0}(\bar{X})$ as the coproduct structure constants of the dual basis $\left\{\left[\xi_{u}\right]\right\}$ in $K_{0}^{T}(X)$ (Lemma 4.2). We then introduce the mixing space $X_{\mathbb{P}}$, which is a bundle over a product of projective spaces $\mathbb{P}$, with fiber $X$. This allows for the reduction from $T$-equivariant $K$-theory to non-equivariant $K$-theory. Using the pairing and duality introduced in the previous section, we realize the structure constants in terms of certain cohomology groups (cf. Lemma 4.10). Finally, we introduce the mixing group $\Gamma$ whose action is sufficient to allow for a transverality result necessary to prove part a) of our main technical result (Theorem 5.1).

Definition 4.1 (Structure constants $d_{u, v}^{w}$ ). By Lemma 3.1, in $K_{T}^{0}(\bar{X})$ we have:

$$
\left[\mathcal{O}_{X^{u}}\right] \cdot\left[\mathcal{O}_{X^{v}}\right]=\sum_{w \in W} d_{u, v}^{w}\left[\mathcal{O}_{X^{w}}\right], \text { for unique } d_{u, v}^{w} \in R(T)
$$

where infinitely many of $d_{u, v}^{w}$ may be non-zero.

Lemma 4.2. Write, in $K_{0}^{T}(X \times X)$ under the diagonal action of $T$ on $X \times X$,

$$
\Delta_{*}\left[\xi_{w}\right]=\sum_{u, v} e_{u, v}^{w}\left[\xi_{u} \otimes \xi_{v}\right], \text { for } e_{u, v}^{w} \in R(T),
$$

where $\Delta: X \rightarrow X \times X$ is the diagonal map. Then, $e_{u, v}^{w}=d_{u, v}^{w}$. 
Proof. Let $\bar{\Delta}: \bar{X} \rightarrow \bar{X} \times \bar{X}$ be the diagonal map, and note that $\bar{\Delta}^{*}\left[\mathcal{O}_{X^{u}} \otimes \mathcal{O}_{X^{v}}\right]=$ $\left[\mathcal{O}_{X^{u}}\right] \cdot\left[\mathcal{O}_{X^{v}}\right]$. Hence, we have:

$$
\begin{aligned}
d_{u, v}^{w} & \left.=\left\langle\sum_{w^{\prime} \in W} d_{u, v}^{w^{\prime}}\left[\mathcal{O}_{X^{w^{\prime}}}\right],\left[\xi_{w}\right]\right\rangle, \text { by Proposition } 3.10\right] \\
& =\left\langle\left[\mathcal{O}_{X^{u}}\right] \cdot\left[\mathcal{O}_{X^{v}}\right],\left[\xi_{w}\right]\right\rangle, \text { by (2) } \\
& =\left\langle\bar{\Delta}^{*}\left[\mathcal{O}_{X^{u}} \otimes \mathcal{O}_{X^{v}}\right],\left[\xi_{w}\right]\right\rangle \\
& =\left\langle\left[\mathcal{O}_{X^{u}} \otimes \mathcal{O}_{X^{v}}\right], \Delta_{*}\left[\xi_{w}\right]\right\rangle \\
& =\left\langle\left[\mathcal{O}_{X^{u}} \otimes \mathcal{O}_{X^{v}}\right], \sum_{u^{\prime}, v^{\prime}} e_{u^{\prime}, v^{\prime}}^{w}\left[\xi_{u^{\prime}} \otimes \xi_{v^{\prime}}\right]\right\rangle, \text { by (3) } \\
& \left.=e_{u, v}^{w}, \text { by Proposition } 3.10\right]
\end{aligned}
$$

We now prepare to define the mixing space. Let $\mathbb{P}:=\left(\mathbb{P}^{N}\right)^{r}$ where $r=\operatorname{dim} T$ and $N$ is some large fixed integer. Let $[N]=\{0,1, \ldots, N\}$ and let $j=\left(j_{1}, j_{2}, \ldots, j_{r}\right) \in$ $[N]^{r}$. We define

$$
\mathbb{P}^{j}:=\mathbb{P}^{N-j_{1}} \times \cdots \times \mathbb{P}^{N-j_{r}}
$$

and similarly define

$$
\mathbb{P}_{j}:=\mathbb{P}^{j_{1}} \times \cdots \times \mathbb{P}^{j_{r}} .
$$

We also define the boundary of $\mathbb{P}_{j}$ by

$$
\partial \mathbb{P}_{j}:=\left(\mathbb{P}^{j_{1}-1} \times \mathbb{P}^{j_{2}} \times \cdots \times \mathbb{P}^{j_{r}}\right) \cup \cdots \cup\left(\mathbb{P}^{j_{1}} \times \cdots \times \mathbb{P}^{j_{r-1}} \times \mathbb{P}^{j_{r}-1}\right),
$$

where we interpret $\mathbb{P}^{-1}:=\emptyset$ to be the empty set. Throughout this paper we will identify $T \simeq\left(\mathbb{C}^{*}\right)^{r}$ via $t \mapsto\left(e^{-\alpha_{1}}(t), \ldots, e^{-\alpha_{r}}(t)\right)$.

Definition 4.3 (Mixing space $\left.X_{\mathbb{P}}\right)$. Let $E(T)_{\mathbb{P}}:=\left(\mathbb{C}^{N+1} \backslash\{0\}\right)^{r}$ be the total space of the standard principal $T$-bundle $E(T)_{\mathbb{P}} \rightarrow \mathbb{P}$. We can view $E(T)_{\mathbb{P}} \rightarrow \mathbb{P}$ as a finite dimensional approximation of the classifying bundle for $T$. Define $X_{\mathbb{P}}:=$ $E(T)_{\mathbb{P}} \times^{T} X$ and let $p: X_{\mathbb{P}} \rightarrow \mathbb{P}$ be the Zariski-locally trivial fibration with fiber $X=G^{\mathrm{min}} / B$.

For any $T$-scheme $V$ we define $V_{\mathbb{P}}:=E(T)_{\mathbb{P}} \times^{T} V$.

For the rest of this paper we use the notation $Y:=X \times X$ and $\bar{Y}:=\bar{X} \times \bar{X}$. Further, we let $Y_{\mathbb{P}}:=E(T)_{\mathbb{P}} \times^{T} Y \simeq X_{\mathbb{P}} \times_{\mathbb{P}} X_{\mathbb{P}}$, where $T$ acts diagonally on $Y$.

Note that for any $u, v \in W,\left(X_{u}\right)_{\mathbb{P}}$ and $\left(X_{u} \times X_{v}\right)_{\mathbb{P}}$ are CM, as they are fiber bundles over $\mathbb{P}$, and hence locally a product of $\mathrm{CM}$ schemes. Thus, they have dualizing sheaves.

Definition 4.4. We define the sheaf $\left(\xi_{u}\right)_{\mathbb{P}}$ on $X_{\mathbb{P}}$ by

$$
\left(\xi_{u}\right)_{\mathbb{P}}:=\left(e^{\rho} \mathscr{L}(\rho)\right)_{\mathbb{P}} \otimes \omega_{\left(X_{u}\right)_{\mathbb{P}}},
$$

where $\left(e^{\rho} \mathscr{L}(\rho)\right)_{\mathbb{P}}$ is defined by

$$
E(T)_{\mathbb{P}} \times^{T} e^{\rho} \mathscr{L}(\rho) \rightarrow X_{\mathbb{P}} .
$$


Proposition 4.5. $K_{0}\left(X_{\mathbb{P}}\right):=$ Limit $_{n \rightarrow \infty} K_{0}\left(\left(X_{n}\right)_{\mathbb{P}}\right)$ is a free module over the ring $K_{0}(\mathbb{P})=K^{0}(\mathbb{P})$ with basis $\left\{\left[\left(\xi_{w}\right)_{\mathbb{P}}\right]\right\}$.

Thus, $K_{0}\left(X_{\mathbb{P}}\right)$ has $\mathbb{Z}$-basis

$$
\left\{p^{*}\left(\left[\mathcal{O}_{\mathbb{P}^{j}}\right]\right) \cdot\left[\left(\xi_{w}\right)_{\mathbb{P}}\right]\right\}_{j \in[N]^{r}, w \in W},
$$

where, as above, $p: X_{\mathbb{P}} \rightarrow \mathbb{P}$ is the projection.

Proof. This follows from [CG, $§ 5.2 .14$ and Theorem 5.4.17] as well as the fact that the transition matrix between the basis $\left\{\left[\mathcal{O}_{X_{w}}\right]\right\}$ and $\left\{\left[\xi_{w}\right]\right\}$ is upper triangular with invertible diagonal entries.

Definition 4.6. We define the sheaf $\widetilde{\xi_{u} \otimes \xi_{v}}$ on $Y_{\mathbb{P}}$ by

$$
\widetilde{\xi_{u} \otimes \xi_{v}}:=\left(e^{2 \rho} \mathscr{L}(\rho) \otimes \mathscr{L}(\rho)\right)_{\mathbb{P}} \otimes \omega_{\left(X_{u} \times X_{v}\right)_{\mathbb{P}}},
$$

where $\left(e^{2 \rho} \mathscr{L}(\rho) \otimes \mathscr{L}(\rho)\right)_{\mathbb{P}}$ is defined by

$$
E(T)_{\mathbb{P}} \times^{T}\left(e^{2 \rho} \mathscr{L}(\rho) \otimes \mathscr{L}(\rho)\right) \rightarrow Y_{\mathbb{P}}
$$

The diagonal map $\Delta: X \rightarrow Y$ gives rise to the embedding $\tilde{\Delta}: X_{\mathbb{P}} \rightarrow Y_{\mathbb{P}}$. Thus, by the previous proposition, we may write

$$
\left.\tilde{\Delta}_{*}\left[\left(\xi_{w}\right)_{\mathbb{P}}\right]=\sum_{u, v \in W, j \in[N]^{r}} c_{u, v}^{w}(j) \hat{p}^{*}\left[\mathcal{O}_{\mathbb{P}^{j}}\right] \cdot \widetilde{\left[\xi_{u} \otimes \xi_{v}\right.}\right] \in K_{0}\left(Y_{\mathbb{P}}\right), \text { for unique } c_{u, v}^{w}(j) \in \mathbb{Z},
$$

where $\hat{p}: Y_{\mathbb{P}} \rightarrow \mathbb{P}$ is the projection.

The following lemma makes precise the reduction from $T$-equivariant $K$-theory of $\bar{X}$ to the ordinary $K$-theory of the mixing space.

Lemma 4.7. For any $u, v, w \in W$ we can choose $N$ large enough and express

$$
d_{u, v}^{w}=\sum_{j \in[N]^{r}} d_{u, v}^{w}(j)\left(e^{-\alpha_{1}}-1\right)^{j_{1}} \ldots\left(e^{-\alpha_{r}}-1\right)^{j_{r}},
$$

where $d_{u, v}^{w}(j) \in \mathbb{Z}$.

Then,

$$
d_{u, v}^{w}(j)=(-1)^{|j|} c_{u, v}^{w}(j), \text { where }|j|:=\sum_{i=1}^{r} j_{i} .
$$

Proof. This follows from Lemma 4.2 and [GK, Lemma 6.2] (see also [AGM, §3]).

Lemma 4.8. For any coherent sheaf $\mathcal{S}$ on $\mathbb{P}$ and any $u, v \in W$ we have:

a) $\mathscr{T}_{i} \operatorname{\mathcal {O}}_{\bar{Y}_{\mathbb{P}}}\left(\bar{p}^{*}(\mathcal{S}), \widetilde{\xi_{u} \otimes \xi_{v}}\right)=0, \forall i>0$,

b) $\mathscr{T}_{i} \operatorname{O}_{\bar{Y}_{\mathbb{P}}}\left(\bar{p}^{*}(\mathcal{S}), \mathcal{O}_{\left(X^{u} \times X^{v}\right)_{\mathbb{P}}}\right)=0, \forall i>0$,

where $\bar{p}: \bar{Y}_{\mathbb{P}} \rightarrow \mathbb{P}$ is the projection.

Proof. As the statements are local in $\mathbb{P}$, we may replace $\bar{Y}_{\mathbb{P}}$ by $\mathcal{U} \times \bar{Y}$, for some open set $\mathcal{U} \subset \mathbb{P}$. Then,

$$
\begin{gathered}
\bar{p}^{*} \mathcal{S} \simeq S \otimes \mathcal{O}_{\bar{Y}}, \\
\widetilde{\xi_{u} \otimes \xi_{v}} \simeq \mathcal{O}_{\mathcal{U}} \otimes\left(\xi_{u} \otimes \xi_{v}\right), \\
\mathcal{O}_{\left(X^{u} \times X^{v}\right)_{\mathbb{P}}} \simeq \mathcal{O}_{\mathcal{U}} \otimes\left(\mathcal{O}_{X^{u}} \otimes \mathcal{O}_{X^{v}}\right) .
\end{gathered}
$$


Applying the above, followed by the Kunneth formula, gives for part a),

$$
\begin{aligned}
& \mathscr{T}_{\text {or }_{i}}^{\mathcal{O}_{\mathcal{U} \times \bar{Y}}}\left(\bar{p}^{*}(\mathcal{S}), \widetilde{\xi_{u} \otimes \xi_{v}}\right)=\mathscr{T}_{\text {or }}^{\mathcal{O}_{i} \otimes \mathcal{O}_{\bar{Y}}}\left(\mathcal{S} \otimes \mathcal{O}_{\bar{Y}}, \mathcal{O}_{\mathcal{U}} \otimes\left(\xi_{u} \otimes \xi_{v}\right)\right) \\
& =\bigoplus_{j+k=i} \mathscr{T}_{o r}{ }_{j}^{\mathcal{O}_{\mathcal{U}}}\left(\mathcal{S}, \mathcal{O}_{\mathcal{U}}\right) \otimes \mathscr{T} r_{k}^{\mathcal{O}_{\bar{Y}}}\left(\mathcal{O}_{\bar{Y}}, \xi_{u} \otimes \xi_{v}\right) \\
& =0, \text { for } i>0 \text {. }
\end{aligned}
$$

A similar computation gives part $\mathrm{b})$.

Lemma 4.9. For any coherent sheaf $\mathcal{S}$ on $\mathbb{P}$ and any $u \in W$ we have:

$$
\mathscr{T}_{\text {or }_{i}}^{\mathcal{O}_{Y_{\mathbb{P}}}}\left(\hat{p}^{*}(\mathcal{S}), \tilde{\Delta}_{*}\left(\left(\xi_{u}\right)_{\mathbb{P}}\right)\right)=0, \forall i>0,
$$

where, as earlier, $\hat{p}: Y_{\mathbb{P}} \rightarrow \mathbb{P}$ is the projection.

Proof. As before, since the statement is local in $\mathbb{P}$, we may replace $Y_{\mathbb{P}}$ by $\mathcal{U} \times Y$, for some open set $\mathcal{U} \subset \mathbb{P}$. Then,

$$
\begin{gathered}
\hat{p}^{*} S \simeq S \otimes \mathcal{O}_{Y}, \\
\tilde{\Delta}_{*}\left(\left(\xi_{u}\right)_{\mathbb{P}}\right)=\mathcal{O}_{\mathcal{U}} \otimes \Delta_{*}\left(\xi_{u}\right) .
\end{gathered}
$$

Now, proceed as in the proof of Lemma 4.8 .

Lemma 4.10. With notation as in (4) we have

$$
c_{u, v}^{w}(j)=\left\langle\left[\mathcal{O}_{\left(X^{u} \times X^{v}\right)_{\mathbb{P}}}\right], \hat{p}^{*}\left[\mathcal{O}_{\mathbb{P}_{j}}\left(-\partial \mathbb{P}_{j}\right)\right] \cdot \tilde{\Delta}_{*}\left(\left(\xi_{w}\right) \mathbb{P}\right)\right\rangle,
$$

where the pairing

$$
\langle,\rangle: K^{0}\left(\bar{Y}_{\mathbb{P}}\right) \otimes K_{0}\left(Y_{\mathbb{P}}\right) \rightarrow \mathbb{Z}
$$

is defined (similar to (10) by

$$
\langle[\mathcal{S}],[\mathcal{F}]\rangle=\sum_{i}(-1)^{i} \chi\left(\bar{Y}_{\mathbb{P}}, \mathscr{T} \text { or }_{i}^{\mathcal{O}_{\bar{Y}}}(\mathcal{S}, \mathcal{F})\right),
$$

where $\chi$ denotes the Euler-Poincaré characteristic, and (as earlier) the map $\hat{p}$ : $Y_{\mathbb{P}} \rightarrow \mathbb{P}$ denotes the projection.

Proof. First, we have

$\left\langle\left[\mathcal{O}_{\left(X^{u} \times X^{v}\right)_{\mathbb{P}}}\right], \hat{p}^{*}\left[\mathcal{O}_{\mathbb{P}_{j}}\left(-\partial \mathbb{P}_{j}\right)\right] \cdot \tilde{\Delta}_{*}\left(\left(\xi_{w}\right)_{\mathbb{P}}\right)\right\rangle=\left\langle\bar{p}^{*}\left[\mathcal{O}_{\mathbb{P}_{j}}\left(-\partial \mathbb{P}_{j}\right)\right] \cdot\left[\mathcal{O}_{\left(X^{u} \times X^{v}\right)_{\mathbb{P}}}\right], \tilde{\Delta}_{*}\left(\left(\xi_{w}\right)_{\mathbb{P}}\right)\right\rangle$, where $\bar{p}: \bar{Y}_{\mathbb{P}} \rightarrow \mathbb{P}$ denotes the projection. To see this, first take a locally free finite resolution of $\mathcal{O}_{\left(X^{u} \times X^{v}\right)_{\mathbb{P}}}$ on a quasi-compact open subset of $\bar{Y}_{\mathbb{P}}$ and a locally free finite resolution of $\mathcal{O}_{\mathbb{P}_{j}}\left(-\partial \mathbb{P}_{j}\right)$ on $\mathbb{P}$. Then, use the fact that for a locally free sheaf $\mathcal{F}$ on a quasi-compact open subset of $\bar{Y}_{\mathbb{P}}$ and a locally free sheaf $\mathcal{G}$ on $\mathbb{P}$, we have

$$
\left\langle\mathcal{F}, \hat{p}^{*}(\mathcal{G}) \cdot \tilde{\Delta}_{*}\left(\left(\xi_{w}\right)_{\mathbb{P}}\right)\right\rangle=\left\langle\bar{p}^{*}(\mathcal{G}) \cdot \mathcal{F}, \tilde{\Delta}_{*}\left(\left(\xi_{w}\right)_{\mathbb{P}}\right)\right\rangle .
$$

Now, the lemma follows from (4), Lemma 4.8, Proposition 3.10 and Ku1, Identity 20].

We now introduce the mixing group $\Gamma$ which acts on $\bar{Y}_{\mathbb{P}}$.

Definition 4.11 (Mixing group $\Gamma$ ). Let $T$ act on $B$ via

$$
t \cdot b=t b t^{-1}
$$

for $t \in T, b \in B$. This action induces a natural action of $\Delta T$ on $B \times B$. Consider the ind-group scheme over $\mathbb{P}$ :

$$
\left(B^{2}\right)_{\mathbb{P}}=E(T)_{\mathbb{P}} \times^{T} B^{2} \rightarrow \mathbb{P} .
$$


Let $\Gamma_{0}$ denote the group of global sections of $\left(B^{2}\right)_{\mathbb{P}}$ under pointwise multiplication. Since $G L(N+1)^{r}$ acts canonically on $\left(B^{2}\right)_{\mathbb{P}}$ in a way compatible with its action on $\mathbb{P}$, it also acts on $\Gamma_{0}$ via inverse pullback. We define the mixing group $\Gamma$ to be the semidirect product $\Gamma:=\Gamma_{0} \rtimes G L(N+1)^{r}$ :

$$
1 \rightarrow \Gamma_{0} \rightarrow \Gamma \rightarrow G L(N+1)^{r} \rightarrow 1 .
$$

By Ku1, Lemmas 4.7 and 4.8 (more precisely, the paragraph following these lemmas)], we have the following two lemmas:

Lemma 4.12. $\Gamma$ is connected.

Lemma 4.13. For any $\bar{e} \in \mathbb{P}$ and any $\left(b, b^{\prime}\right)$ in the fiber of $\left(B^{2}\right)_{\mathbb{P}}$ over $\bar{e}$ there exists a section $\gamma \in \Gamma_{0}$ such that $\gamma(\bar{e})=\left(b, b^{\prime}\right)$.

We define the action of $\Gamma$ on $\bar{Y}_{\mathbb{P}}$ via

$$
(\gamma, g) \cdot[e,(x, y)]=[g e, \gamma(g e) \cdot(x, y)]
$$

for $\gamma \in \Gamma_{0}, g \in G L(N+1)^{r}, e \in E(T)_{\mathbb{P}}$, and $(x, y) \in \bar{Y}$, where the action of $\Gamma_{0}$ is via the standard action of $B \times B$ on $\bar{Y}=\bar{X}^{2}$. It follows from Lemma 4.13 that the orbits of the $\Gamma$-action on $\bar{Y}_{\mathbb{P}}$ are precisely equal to $\left\{\left(C_{w} \times C_{w^{\prime}}\right)_{\mathbb{P}}\right\}$.

\section{Statement of MAin RESUlts}

We now come to our main technical result.

Theorem 5.1. For general $\gamma \in \Gamma$ and any $u, v, w \in W, j \in[N]^{r}$ we have:

a) For all $i>0$,

$$
\mathscr{T}_{i} \boldsymbol{\mathcal { O }}_{\bar{Y}_{\mathbb{P}}}\left(\gamma_{*} \mathcal{O}_{\left(X^{u} \times X^{v}\right)_{\mathbb{P}}}, \hat{p}^{*}\left(\mathcal{O}_{\mathbb{P}_{j}}\left(-\partial \mathbb{P}_{j}\right)\right) \otimes \mathcal{O}_{Y_{\mathbb{P}}} \tilde{\Delta}_{*}\left(\left(\xi_{w}\right)_{\mathbb{P}}\right)\right)=0
$$

b) Assume $c_{u, v}^{w}(j) \neq 0$. For all $p \neq|j|+\ell(w)-\ell(u)-\ell(v)$,

$$
H^{p}\left(\bar{Y}_{\mathbb{P}}, \gamma_{*} \mathcal{O}_{\left(X^{u} \times X^{v}\right)_{\mathbb{P}}} \otimes_{\mathcal{O}_{\bar{Y}_{\mathbb{P}}}}\left(\hat{p}^{*}\left(\mathcal{O}_{\mathbb{P}_{j}}\left(-\partial \mathbb{P}_{j}\right)\right) \otimes_{\mathcal{O}_{Y_{\mathbb{P}}}} \tilde{\Delta}_{*}\left(\left(\xi_{w}\right)_{\mathbb{P}}\right)\right)\right)=0
$$

where $|j|:=\sum_{i=1}^{r} j_{i}$.

Proof. Deferred to the later sections. Part a) is proved in Section 6, while part b) is proved in Section 9.

Since $\Gamma$ is connected, Lemmas 4.10, 4.9, and Theorem 5.1 together give:

Corollary 5.2. $(-1)^{\ell(w)-\ell(u)-\ell(v)+|j|} c_{u, v}^{w}(j) \in \mathbb{Z}_{\geq 0}$.

As an immediate consequence of Corollary 5.2 and Lemma 4.7 we get:

Theorem 5.3. For any symmetrizable Kac-Moody group $G$ and any $u, v, w \in W$, the structure constants of the product in the basis $\left\{\left[\mathcal{O}_{X^{w}}\right]\right\}$ in $K_{T}^{0}(\bar{X})$ satisfy

$$
(-1)^{\ell(w)+\ell(u)+\ell(v)} d_{u, v}^{w} \in \mathbb{Z}_{\geq 0}\left[\left(e^{-\alpha_{1}}-1\right), \ldots,\left(e^{-\alpha_{r}}-1\right)\right] .
$$




\section{Proof of part a) of Theorem 5.1}

The key tool used to prove part a) of Theorem 5.1 is the following transversality result, taken from [AGM, Theorem 2.3] (originally due to Sierra).

Theorem 6.1. Let $X$ be a variety with a left action of an algebraic group $G$ and let $\mathcal{F}$ be a coherent sheaf on $X$. Suppose that $\mathcal{F}$ is homologically transverse to the closures of the $G$-orbits on $X$. Then, for each coherent sheaf $\mathcal{E}$ on $X$, there is a Zariski-dense open set $U \subseteq G$ such that $\mathscr{T}^{\text {or }}{ }_{j}^{\mathcal{O}_{X}}\left(\mathcal{F}, g_{*} \mathcal{E}\right)=0$ for all $j \geq 1$ and all $g \in U$.

Theorem 5.1 part a) is a particular case of the following slightly more general result by taking $\mathcal{E}=\hat{p}^{*}\left(\mathcal{O}_{\mathbb{P}_{j}}\left(-\partial \mathbb{P}_{j}\right)\right) \otimes_{\mathcal{O}_{Y_{\mathbb{P}}}} \tilde{\Delta}_{*}\left(\left(\xi_{w}\right)_{\mathbb{P}}\right)$.

Theorem 6.2. Let $w \in W$ and let $\mathcal{E}$ be a coherent sheaf on $\left(Y_{w}\right)_{\mathbb{P}}:=\left(X_{w} \times X_{w}\right)_{\mathbb{P}}$. Then, for general $\gamma \in \Gamma$ and any $u, v \in W$ we have:

$$
\mathscr{T}_{\text {or }}^{\mathcal{O}_{\bar{Y}_{\mathbb{P}}}}\left(\gamma_{*} \mathcal{O}_{\left(X^{u} \times X^{v}\right)_{\mathbb{P}}}, \mathcal{E}\right)=0, \forall i>0 .
$$

Proof. We first show that for any $\gamma \in \Gamma$,

$$
\mathscr{T}_{o r} \boldsymbol{O}_{\bar{Y}_{\mathbb{P}}}\left(\mathcal{O}_{\left(X^{u} \times X^{v}\right)_{\mathbb{P}}}, \gamma_{*} \mathcal{E}\right)=\mathscr{T} \operatorname{or}_{i}^{\mathcal{O}_{\left(Y_{w}\right)_{\mathbb{P}}}}\left(\mathcal{O}_{\left(Y_{w}\right)_{\mathbb{P}}} \otimes \mathcal{O}_{\bar{Y}_{\mathbb{P}}} \mathcal{O}_{\left(X^{u} \times X^{v}\right)_{\mathbb{P}}}, \gamma_{*} \mathcal{E}\right) .
$$

Since $\gamma_{*} \mathcal{E}$ is a coherent sheaf on $\left(Y_{w}\right)_{\mathbb{P}}$, we can replace $\gamma_{*} \mathcal{E}$ by $\mathcal{E}$ itself.

As the assertion is local on $\mathbb{P}$ we may assume $\bar{Y}_{\mathbb{P}} \simeq \mathbb{P} \times \bar{Y}$ and that

$$
\begin{gathered}
\mathcal{O}_{\left(X^{u} \times X^{v}\right)_{\mathbb{P}}} \simeq \mathcal{O}_{\mathbb{P}} \otimes\left(\mathcal{O}_{X^{u}} \otimes \mathcal{O}_{X^{v}}\right), \\
\mathcal{O}_{\left(Y_{w}\right)_{\mathbb{P}}} \simeq \mathcal{O}_{\mathbb{P}} \otimes\left(\mathcal{O}_{X_{w}} \otimes \mathcal{O}_{X_{w}}\right) .
\end{gathered}
$$

To simplify notation let $A=\mathcal{O}_{\overline{Y_{\mathbb{P}}}}, B=\mathcal{O}_{\left(Y_{w}\right)_{\mathbb{P}}}, M=\mathcal{O}_{\left(X^{u} \times X^{v}\right)_{\mathbb{P}}}$ and $N=\mathcal{E}$. Take an $A$-free resolution $\mathcal{F}_{\bullet} \rightarrow M$ on an open subset of $\bar{Y}_{\mathbb{P}}$ of the form $\left(V^{u^{\prime}} \times V^{v^{\prime}}\right)_{\mathbb{P}}$, where $V^{u}$ is defined as in Lemma 3.3. Then, the homology of the chain complex $B \otimes_{A} \mathcal{F}_{\bullet}$ is by definition $\mathscr{T}_{o r}^{A}(M, B)$. Moreover,

$$
\mathscr{T}_{i}^{A}(M, B)=0, \forall i>0 \text {. }
$$

Indeed, locally on $\mathbb{P}$,

$$
\begin{aligned}
& \mathscr{T}_{\text {or }}^{A}(M, B)=\mathscr{T} \text { or }_{i}^{\mathcal{O}_{\mathbb{P}} \otimes \mathcal{O}_{\bar{Y}}}\left(\mathcal{O}_{\mathbb{P}} \otimes \mathcal{O}_{\left(X^{u} \times X^{v}\right)}, \mathcal{O}_{\mathbb{P}} \otimes \mathcal{O}_{\left(X_{w} \times X_{w}\right)}\right) \\
& =\bigoplus_{j+k=i} \mathscr{T}_{\text {or }}^{\mathcal{O}_{j}}\left(\mathcal{O}_{\mathbb{P}}, \mathcal{O}_{\mathbb{P}}\right) \otimes \mathscr{T}_{\text {or }}{ }_{k}^{\mathcal{O}_{\bar{Y}}}\left(\mathcal{O}_{X^{u} \times X^{v}}, \mathcal{O}_{X_{w} \times X_{w}}\right) \\
& =0, \forall i>0
\end{aligned}
$$

by Lemma 3.7 and the Kunneth formula. Hence, $B \otimes_{A} \mathcal{F}_{\bullet}$ is a $B$-free resolution of $B \otimes_{A} M$.

Thus, the homology of the chain complex $N \otimes_{B}\left(B \otimes_{A} \mathcal{F}_{\bullet}\right)$ is equal to $\mathscr{T}_{\bullet}{ }_{\bullet}\left(B \otimes_{A}\right.$ $M, N)$; but,

$$
N \otimes_{B}\left(B \otimes_{A} \mathcal{F}_{\bullet}\right)=\left(N \otimes_{B} B\right) \otimes_{A} \mathcal{F}_{\bullet}=N \otimes_{A} \mathcal{F}_{\bullet},
$$

so the homology is also equal to $\mathscr{T}_{\bullet}^{A}(M, N)$. Hence,

$$
\mathscr{T}_{\text {or }}^{B}\left(B \otimes_{A} M, N\right)=\mathscr{T}_{\text {or }}^{A}(M, N)
$$

as desired. This proves (5). 
Now, by Lemma 4.13, the closures of the $\Gamma$-orbits of $\left(Y_{w}\right)_{\mathbb{P}}$ are precisely $\left(X_{x} \times\right.$ $\left.X_{y}\right)_{\mathbb{P}}$, where $x, y \leq w$. Equation (5) implies that the sheaf $\mathcal{F}$ defined by

$$
\mathcal{F}:=\mathcal{O}_{\left(Y_{w}\right)_{\mathbb{P}}} \otimes_{\mathcal{O}_{\bar{Y}}} \mathcal{O}_{\left(X^{u} \times X^{v}\right)_{\mathbb{P}}}
$$

is homologically transverse to the $\Gamma$-orbit closures in $\left(Y_{w}\right)_{\mathbb{P}}$. Indeed, since $\mathcal{O}_{\left(X_{x} \times X_{y}\right)_{\mathbb{P}}}$ is a coherent $\mathcal{O}_{\left(Y_{w}\right) \mathbb{P}}$-module when $x, y \leq w$, equation (5) gives

$$
\begin{aligned}
\mathscr{T}_{i} \boldsymbol{\mathcal { O }}_{\left(Y_{w}\right)_{\mathbb{P}}}\left(\mathcal{F}, \mathcal{O}_{\left(X_{x} \times X_{y}\right)_{\mathbb{P}}}\right) & =\mathscr{T}^{\mathcal{o r}_{i}} \mathcal{O}_{\bar{Y}_{\mathbb{P}}}\left(\mathcal{O}_{\left(X^{u} \times X^{v}\right)_{\mathbb{P}}}, \mathcal{O}_{\left(X_{x} \times X_{y}\right)_{\mathbb{P}}}\right) \\
& =0, \forall i>0
\end{aligned}
$$

by Lemma 3.7 and the Kunneth formula.

Thus, by Theorem 6.1 (with $G=\Gamma, X=\left(Y_{w}\right)_{\mathbb{P}}$, and $\mathcal{E}$ and $\mathcal{F}$ as above), we conclude that for general $\gamma \in \Gamma$,

$$
\mathscr{T} \operatorname{or}_{i}^{\mathcal{O}_{\left(Y_{w}\right)_{\mathbb{P}}}}\left(\mathcal{O}_{\left(Y_{w}\right)_{\mathbb{P}}} \otimes \mathcal{O}_{\bar{Y}_{\mathbb{P}}} \mathcal{O}_{\left(X^{u} \times X^{v}\right)_{\mathbb{P}}}, \gamma_{*} \mathcal{E}\right)=0, \forall i>0 .
$$

Here we note that although $\Gamma$ is infinite dimensional, the action of $\Gamma$ on $\left(Y_{w}\right)_{\mathbb{P}}$ factors through the action of a finite dimensional quotient group of $\Gamma$.

Now, (5) gives

$$
\mathscr{T}_{\text {or }} \mathcal{O}_{\bar{Y}_{\mathbb{P}}}\left(\mathcal{O}_{\left(X^{u} \times X^{v}\right)_{\mathbb{P}}}, \gamma_{*} \mathcal{E}\right)=0, \forall i>0
$$

which is equivalent to the desired vanishing.

\section{The SCHEMES $\mathcal{Z}$ AND $\partial \mathcal{Z}$}

For $u, v \leq w$ we use the notation $X_{w}^{u, v}:=X_{w}^{u} \times X_{w}^{v}$. We also write $X_{w}^{2}:=$ $X_{w} \times X_{w}$. For any $j \in[N]^{r}$, we let $\left(X_{w}\right)_{j}$ denote the inverse image of $\mathbb{P}_{j}$ through the map $E(T)_{\mathbb{P}} \times^{T} X_{w} \rightarrow \mathbb{P}$.

Similarly, for $u, v \leq w$ we write $Z_{w}^{u, v}:=Z_{w}^{u} \times Z_{w}^{v}$, where $Z_{w}^{u}$ is the $T$-equivariant desingularization of $X_{w}^{u}$ as in [Ku1, Theorem 6.8]. We also write $Z_{w}^{2}:=Z_{w} \times Z_{w}$, where $Z_{w}$ is a BSDH variety as in $[\mathrm{Ku} 2, \S 7.1 .3]$. For any $j \in[N]^{r}$, we let $\left(Z_{w}\right)_{j}$ denote the inverse image of $\mathbb{P}_{j}$ through the map $E(T)_{\mathbb{P}} \times^{T} Z_{w} \rightarrow \mathbb{P}$.

The action of $B$ on $Z_{w}$ factors through the action of a finite dimensional quotient group $\bar{B}$ containing the maximal torus $H$. Further, the action of $\Gamma$ on $\left(X_{w}^{2}\right)_{\mathbb{P}}$ descends to an action of the finite dimensional quotient group

$$
\bar{\Gamma}:=\bar{\Gamma}_{0} \rtimes G L(N+1)^{r},
$$

where $\bar{\Gamma}_{0}$ is the group of global sections of the bundle $E(T)_{\mathbb{P}} \times^{T}\left(\bar{B}^{2}\right) \rightarrow \mathbb{P}$.

From [Ku1, Lemmas 6.11 and 6.12] we have

Lemma 7.1. Let $u, v \leq w$. The map

$$
m: \bar{\Gamma} \times\left(X_{w}^{u, v}\right)_{\mathbb{P}} \rightarrow\left(X_{w}^{2}\right)_{\mathbb{P}}, m(\gamma, x)=\gamma \cdot \pi_{2}(x),
$$

is flat, where $\pi_{2}:\left(X_{w}^{u} \times X_{w}^{v}\right)_{\mathbb{P}} \rightarrow\left(X_{w}^{2}\right)_{\mathbb{P}}$ is induced from the canonical map $X_{w}^{u} \times$ $X_{w}^{v} \rightarrow X_{w}^{2}$.

Lemma 7.2. Let $u, v \leq w$. The map

$$
\tilde{m}: \bar{\Gamma} \times\left(Z_{w}^{u, v}\right)_{\mathbb{P}} \rightarrow\left(Z_{w}^{2}\right)_{\mathbb{P}}, \tilde{m}(\gamma, x)=\gamma \cdot \tilde{\pi}_{2}(x),
$$

is smooth, where $\tilde{\pi}_{2}:\left(Z_{w}^{u} \times Z_{w}^{v}\right)_{\mathbb{P}} \rightarrow\left(Z_{w}^{2}\right)_{\mathbb{P}}$ is induced from the canonical map $Z_{w}^{u} \times Z_{w}^{v} \rightarrow Z_{w}^{2}$. 
Define $\mathcal{Z}$ to be the fiber product

$$
\mathcal{Z}:=\left(\bar{\Gamma} \times\left(X_{w}^{u, v}\right)_{\mathbb{P}}\right) \times_{\left(X_{w}^{2}\right)_{\mathbb{P}}} \tilde{\Delta}\left(\left(X_{w}\right)_{j}\right)
$$

and $\widetilde{\mathcal{Z}}$ to be the fiber product

$$
\widetilde{\mathcal{Z}}:=\left(\bar{\Gamma} \times\left(Z_{w}^{u, v}\right)_{\mathbb{P}}\right) \times\left(Z_{w}^{2}\right)_{\mathbb{P}} \tilde{\Delta}\left(\left(Z_{w}\right)_{j}\right)
$$

as in the commutative diagram:

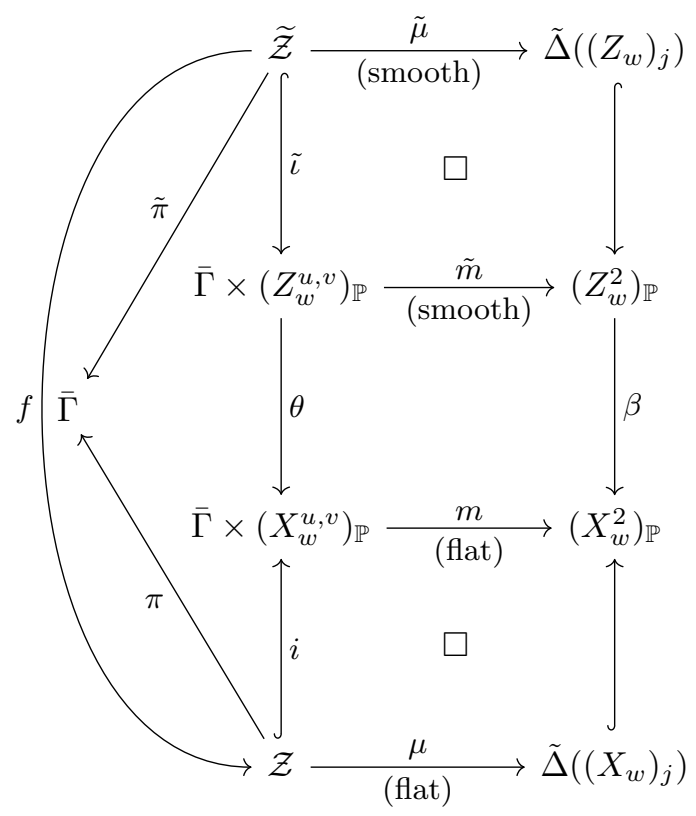

In the above diagram, $\square$ denotes a fiber square. Note that the maps $\theta$ and $\beta$ above are desingularizations. The maps $\pi: \mathcal{Z} \rightarrow \bar{\Gamma}$ and $\tilde{\pi}: \widetilde{\mathcal{Z}} \rightarrow \bar{\Gamma}$ are induced by the projections onto the first factor. The map $f: \widetilde{\mathcal{Z}} \rightarrow \mathcal{Z}$ is defined by $f:=\theta \circ \tilde{\iota}$. It is clear that the image of $f$ is indeed contained inside of $\mathcal{Z}$ using commutativity of the diagram, along with the fact that $\left.\beta\left(\tilde{\Delta}\left(\left(Z_{w}\right)_{j}\right)\right)=\tilde{\Delta}\left(\left(X_{w}\right)_{j}\right)\right)$.

We define the boundary of $\left(X_{w}\right)_{j}$ by

$$
\partial\left(\left(X_{w}\right)_{j}\right):=\left(\partial X_{w}\right)_{j} \cup\left(X_{w}\right)_{\partial \mathbb{P}_{j}}
$$

and similarly define the boundary of $\left(Z_{w}\right)_{j}$ by

$$
\partial\left(\left(Z_{w}\right)_{j}\right):=\left(\partial Z_{w}\right)_{j} \cup\left(Z_{w}\right)_{\partial \mathbb{P}_{j}},
$$

where $\partial Z_{w}:=\varphi^{-1}\left(\partial X_{w}\right)$ and $\varphi: Z_{w} \rightarrow X_{w}$ denotes the desingularization.

We define the boundary of $\mathcal{Z}$ by

$$
\partial \mathcal{Z}:=\left(\bar{\Gamma} \times\left(X_{w}^{u, v}\right)_{\mathbb{P}}\right) \times_{\left(X_{w}^{2}\right)_{\mathbb{P}}} \tilde{\Delta}\left(\partial\left(\left(X_{w}\right)_{j}\right)\right)
$$

and similarly define the boundary of $\tilde{\mathcal{Z}}$ by

$$
\partial \tilde{\mathcal{Z}}:=\left(\bar{\Gamma} \times\left(Z_{w}^{u, v}\right)_{\mathbb{P}}\right) \times{ }_{\left(Z_{w}^{2}\right)_{\mathbb{P}}} \tilde{\Delta}\left(\partial\left(\left(Z_{w}\right)_{j}\right)\right) .
$$

Observe that $f^{-1}(\partial \mathcal{Z})=\partial \widetilde{\mathcal{Z}}$ is the scheme-theoretic inverse image. 
We will need the following lemmas, which are restatements of [Ku1, Lemmas 7.2 and 7.3], respectively, ([Ku1, Lemma 7.3] is originally from [FP, Lemma on page $108])$.

Lemma 7.3. Let $f: W \rightarrow X$ be a flat morphism from a pure-dimensional $\mathrm{CM}$ scheme $W$ of finite type over $\mathbb{C}$ to a $\mathrm{CM}$ irreducible variety $X$ and let $Y$ be a closed CM subscheme of $X$ of pure codimension d. Set $Z:=f^{-1}(Y)$. If $\operatorname{codim}_{W}(Z) \geq d$, then equality holds and $Z$ is $\mathrm{CM}$.

Lemma 7.4. Let $f: W \rightarrow X$ be a morphism from a pure-dimensional CM scheme $W$ of finite type over $\mathbb{C}$ to a smooth irreducible variety $X$ and let $Y$ be a closed $\mathrm{CM}$ subscheme of $X$ of pure codimension d. Set $Z:=f^{-1}(Y)$. If $\operatorname{codim}_{W}(Z) \geq d$, then equality holds and $Z$ is CM.

Proposition 7.5. The scheme $\mathcal{Z}$ is normal, irreducible, and $\mathrm{CM}$, of dimension

$$
\operatorname{dim} \mathcal{Z}=|j|+\ell(w)-\ell(u)-\ell(v)+\operatorname{dim} \bar{\Gamma} .
$$

The scheme $\widetilde{\mathcal{Z}}$ is irreducible, and the map $f: \widetilde{\mathcal{Z}} \rightarrow \mathcal{Z}$ is a proper birational map. Hence, $\widetilde{\mathcal{Z}}$ is a desingularization of $\mathcal{Z}$.

Proof. By [H, Chapter III, Corollary 9.6], the fibers of $m$ are pure of dimension

$$
\operatorname{dim} \bar{\Gamma}+\operatorname{dim}\left(X_{w}^{u, v}\right)_{\mathbb{P}}-\operatorname{dim}\left(X_{w}^{2}\right)_{\mathbb{P}} .
$$

Since the fibers of $\mu$ are the same as those of $m$, applying loc. cit. to $\mu$ gives that $\mathcal{Z}$ is pure of dimension

$$
\begin{gathered}
\operatorname{dim} \mathcal{Z}=\operatorname{dim} \bar{\Gamma}+\operatorname{dim}\left(X_{w}^{u, v}\right)_{\mathbb{P}}-\operatorname{dim}\left(X_{w}^{2}\right)_{\mathbb{P}}+\operatorname{dim} \tilde{\Delta}\left(\left(X_{w}\right)_{j}\right) \\
=|j|+\ell(w)-\ell(u)-\ell(v)+\operatorname{dim} \bar{\Gamma}
\end{gathered}
$$

The remainder of the proof of the proposition follows from the proof of $\mathrm{Ku} 1$, Proposition 7.4 and Lemma 7.5] (Note that there is a slight difference in the definition of $\mathcal{Z}$ and $\widetilde{\mathcal{Z}}$ between ours and that in $[\mathrm{Ku} 1$.) Further, $\widetilde{\mathcal{Z}}$ is non-singular since $\tilde{\mu}$ is a smooth morphism with non-singular base.

Lemma 7.6. Let $G$ be a group acting on a set $X$ and let $Y \subset X$. Consider the action map $m: G \times Y \rightarrow X$. For $x \in X$ denote the orbit of $x$ by $O(x)$ and the stabilizer by $\operatorname{Stab}(x)$. Then, $\operatorname{Stab}(x)$ acts on the fiber $m^{-1}(x)$ and $\operatorname{Stab}(x) \backslash m^{-1}(x) \simeq$ $O(x) \cap Y$.

Proof. It is easy to check that

$$
m^{-1}(x)=\left\{\left(g, h^{-1} x\right): h \in G, h^{-1} x \in Y, g \in \operatorname{Stab}(x) \cdot h\right\} .
$$

Thus, $\operatorname{Stab}(x)$ acts on $m^{-1}(x)$ by left multiplication on the left component. Since every element of $O(x) \cap Y$ is of the form $h^{-1} x$ for some $h \in G$, the second projection $m^{-1}(x) \rightarrow O(x) \cap Y$ is surjective. This map clearly factors through the quotient to give a map $\operatorname{Stab}(x) \backslash m^{-1}(x) \rightarrow O(x) \cap Y$. To show this induced map is injective, note first that each class has a representative of the form $\left(h, h^{-1} x\right)$. Now, if $\left(h_{1}, h_{1}^{-1} x\right)$ and $\left(h_{2}, h_{2}^{-1} x\right)$ satisfy $h_{1}^{-1} x=h_{2}^{-1} x$, then $h_{2} h_{1}^{-1} x=x$, i.e., $h_{2} h_{1}^{-1} \in \operatorname{Stab}(x)$, i.e., $h_{2} \in \operatorname{Stab}(x) \cdot h_{1}$, i.e., $\left(h_{1}, h_{1}^{-1} x\right)$ and $\left(h_{2}, h_{2}^{-1} x\right)$ belong to the same class. 
Proposition 7.7. The scheme $\mathcal{Z}$ has rational singularities.

Proof. Since $\mu$ is flat and $\tilde{\Delta}\left(\left(X_{w}\right)_{j}\right)$ has rational singularities, by [El, Théorème 5$]$ it is sufficient to show that the fibers of $\mu$ are disjoint unions of irreducible varieties with rational singularities (in fact, the fibers of $\mu$ are irreducible, but we do not provide a proof here as we do not need this fact).

Let $x \in \tilde{\Delta}\left(\left(C_{w^{\prime}}\right)_{j}\right)$, where $w^{\prime} \leq w$. Then, by Lemmas 7.6 and 4.13 , we have $\operatorname{Stab}(x) \backslash \mu^{-1}(x) \simeq\left(X^{u} \cap C_{w^{\prime}} \times X^{v} \cap C_{w^{\prime}}\right)_{\mathbb{P}}$, where $\operatorname{Stab}(x)$ is taken with respect to the action of $\bar{\Gamma}$ on $\left(X_{w}^{2}\right)_{\mathbb{P}}$. By $[$ Se, Proposition $3, \S 2.5]$, the quotient map $\bar{\Gamma} \rightarrow$ $\operatorname{Stab}(x) \backslash \bar{\Gamma}$ is locally trivial in the étale topology.

Consider the pullback diagram:

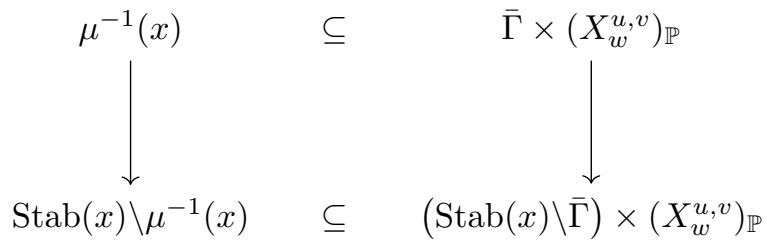

Since the right vertical map is a locally trivial fibration in the étale topology, the left vertical map is too. Now, $\operatorname{Stab}(x) \backslash \mu^{-1}(x) \simeq\left(X^{u} \cap C_{w^{\prime}} \times X^{v} \cap C_{w^{\prime}}\right)_{\mathbb{P}}$ has rational singularities by [KuS, Theorem 3.1]. Further, $\operatorname{Stab}(x)$ being smooth and $\mu^{-1}(x) \rightarrow \operatorname{Stab}(x) \backslash \mu^{-1}(x)$ being locally trivial in the étale topology, we get that $\mu^{-1}(x)$ is a disjoint union of irreducible varieties with rational singularities by [KM, Corollary 5.11].

Proposition 7.8. The scheme $\partial \mathcal{Z}$ is pure of codimension 1 in $\mathcal{Z}$ and is $\mathrm{CM}$.

Proof. Using the fact that $\tilde{\Delta}\left(\left(X_{w}\right)_{\partial \mathbb{P}_{j}}\right)$ and $\tilde{\Delta}\left(\left(\partial X_{w}\right)_{j}\right)$ are equidimensional, applying $[\mathrm{H}$, Chapter III, Corollary 9.6] to their irreducible components gives that $\left(\bar{\Gamma} \times\left(X_{w}^{u, v}\right)_{\mathbb{P}}\right) \times_{\left(X_{w}^{2}\right)_{\mathbb{P}}} \tilde{\Delta}\left(\left(X_{w}\right)_{\partial \mathbb{P}_{j}}\right)$ and $\left(\bar{\Gamma} \times\left(X_{w}^{u, v}\right)_{\mathbb{P}}\right) \times_{\left(X_{w}^{2}\right)_{\mathbb{P}}} \tilde{\Delta}\left(\left(\partial X_{w}\right)_{j}\right)$ are both pure of dimension

$$
\operatorname{dim} \bar{\Gamma}+\operatorname{dim}\left(X_{w}^{u, v}\right)_{\mathbb{P}}-\operatorname{dim}\left(X_{w}^{2}\right)_{\mathbb{P}}+\operatorname{dim} \tilde{\Delta}\left(\left(X_{w}\right)_{j}\right)-1 .
$$

Hence, $\partial \mathcal{Z}$ is pure of codimension 1 in $\mathcal{Z}$.

A similar argument also gives that $\left(\bar{\Gamma} \times\left(X_{w}^{u, v}\right)_{\mathbb{P}}\right) \times_{\left(X_{w}^{2}\right){ }_{\mathbb{P}}} \tilde{\Delta}\left(\left(\partial X_{w}\right)_{\partial \mathbb{P}_{j}}\right)$ is pure of dimension

$$
\operatorname{dim} \bar{\Gamma}+\operatorname{dim}\left(X_{w}^{u, v}\right)_{\mathbb{P}}-\operatorname{dim}\left(X_{w}^{2}\right)_{\mathbb{P}}+\operatorname{dim} \tilde{\Delta}\left(\left(X_{w}\right)_{j}\right)-2 .
$$

Next, we show that $\partial \mathcal{Z}$ is CM. Since $\left(X_{w}\right)_{j}$ is a locally trivial fibration over $\mathbb{P}_{j}$, it is locally a product of $\mathrm{CM}$ schemes and hence is $\mathrm{CM}$. Also, since $\partial \mathbb{P}_{j}$ is $\mathrm{CM}$, we similarly have that $\left(X_{w}\right)_{\partial \mathbb{P}_{j}}$ and hence $\tilde{\Delta}\left(\left(X_{w}\right)_{\partial \mathbb{P}_{j}}\right)$ is CM. Now, applying Lemma 7.3 to $\mu: \mathcal{Z} \rightarrow \tilde{\Delta}\left(\left(X_{w}\right)_{j}\right)$ gives that $\left(\bar{\Gamma} \times\left(X_{w}^{u, v}\right)_{\mathbb{P}}\right) \times_{\left(X_{w}^{2}\right)_{\mathbb{P}}} \tilde{\Delta}\left(\left(X_{w}\right)_{\partial \mathbb{P}_{j}}\right)$ is CM, since $\tilde{\Delta}\left(\left(X_{w}\right)_{\partial \mathbb{P}_{j}}\right)$ and $\mu^{-1}\left(\tilde{\Delta}\left(\left(X_{w}\right)_{\partial \mathbb{P}_{j}}\right)\right)=\left(\bar{\Gamma} \times\left(X_{w}^{u, v}\right)_{\mathbb{P}}\right) \times_{\left(X_{w}^{2}\right)_{\mathbb{P}}} \tilde{\Delta}\left(\left(X_{w}\right)_{\partial \mathbb{P}_{j}}\right)$ are of pure codimension 1 in $\tilde{\Delta}\left(\left(X_{w}\right)_{j}\right)$ and $\mathcal{Z}$, respectively.

Observe that $\partial X_{w}$ is CM. To prove this, use Proposition 3.6 and the argument as in the proof of [Ku1, Corollary 10.5] by taking an embedding of $X_{w}$ into a smooth projective variety. Thus, $\left(\partial X_{w}\right)_{j}$ is locally a product of CM schemes and hence is $\mathrm{CM}$, and hence so is $\tilde{\Delta}\left(\left(\partial X_{w}\right)_{j}\right)$. Now, Lemma 7.3 applied to $\mu: \mathcal{Z} \rightarrow \tilde{\Delta}\left(\left(X_{w}\right)_{j}\right)$ gives that

$$
\left(\bar{\Gamma} \times\left(X_{w}^{u, v}\right)_{\mathbb{P}}\right) \times_{\left(X_{w}^{2}\right)_{\mathbb{P}}} \tilde{\Delta}\left(\left(\partial X_{w}\right)_{j}\right)
$$


is CM since $\tilde{\Delta}\left(\left(\partial X_{w}\right)_{j}\right)$ and $\mu^{-1}\left(\tilde{\Delta}\left(\left(\partial X_{w}\right)_{j}\right)\right)=\left(\bar{\Gamma} \times\left(X_{w}^{u, v}\right)_{\mathbb{P}}\right) \times{ }_{\left(X_{w}^{2}\right)_{\mathbb{P}}} \tilde{\Delta}\left(\left(\partial X_{w}\right)_{j}\right)$ are of pure codimension 1 in $\tilde{\Delta}\left(\left(X_{w}\right)_{j}\right)$ and $\mathcal{Z}$, respectively.

The intersection

$$
\begin{gathered}
\left(\bar{\Gamma} \times\left(X_{w}^{u, v}\right)_{\mathbb{P}}\right) \times_{\left(X_{w}^{2}\right)_{\mathbb{P}}} \tilde{\Delta}\left(\left(\partial X_{w}\right)_{\partial \mathbb{P}_{j}}\right) \\
=\left(\left(\bar{\Gamma} \times\left(X_{w}^{u, v}\right)_{\mathbb{P}}\right) \times_{\left(X_{w}^{2}\right)_{\mathbb{P}}} \tilde{\Delta}\left(\left(X_{w}\right)_{\partial \mathbb{P}_{j}}\right)\right) \cap\left(\left(\bar{\Gamma} \times\left(X_{w}^{u, v}\right)_{\mathbb{P}}\right) \times_{\left(X_{w}^{2}\right)_{\mathbb{P}}} \tilde{\Delta}\left(\left(\partial X_{w}\right)_{j}\right)\right)
\end{gathered}
$$

is of pure codimension 1 in both $\left(\bar{\Gamma} \times\left(X_{w}^{u, v}\right)_{\mathbb{P}}\right) \times\left(X_{w}^{2}\right)_{\mathbb{P}} \tilde{\Delta}\left(\left(X_{w}\right)_{\partial \mathbb{P}_{j}}\right)$ and $\left(\bar{\Gamma} \times\left(X_{w}^{u, v}\right)_{\mathbb{P}}\right)$

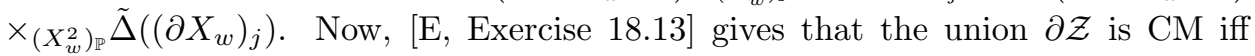
the intersection $\left(\bar{\Gamma} \times\left(X_{w}^{u, v}\right)_{\mathbb{P}}\right) \times_{\left(X_{w}^{2}\right)_{\mathbb{P}}} \tilde{\Delta}\left(\left(\partial X_{w}\right)_{\partial \mathbb{P}_{j}}\right)$ is. But Lemma 7.3 applied to $\mu: \mathcal{Z} \rightarrow \tilde{\Delta}\left(\left(X_{w}\right)_{j}\right)$ gives that $\left(\bar{\Gamma} \times\left(X_{w}^{u, v}\right)_{\mathbb{P}}\right) \times_{\left(X_{w}^{2}\right)_{\mathbb{P}}} \tilde{\Delta}\left(\left(\partial X_{w}\right)_{\partial \mathbb{P}_{j}}\right)$ is CM, since $\tilde{\Delta}\left(\left(\partial X_{w}\right)_{\partial \mathbb{P}_{j}}\right)$ and $\mu^{-1}\left(\tilde{\Delta}\left(\left(\partial X_{w}\right)_{\partial \mathbb{P}_{j}}\right)\right)=\left(\bar{\Gamma} \times\left(X_{w}^{u, v}\right)_{\mathbb{P}}\right) \times_{\left(X_{w}^{2}\right)_{\mathbb{P}}} \tilde{\Delta}\left(\left(\partial X_{w}\right)_{\partial \mathbb{P}_{j}}\right)$ are of pure codimension 2 in $\tilde{\Delta}\left(\left(X_{w}\right)_{j}\right)$ and $\mathcal{Z}$, respectively.

Lemma 7.9. The morphism $\mu: \mathcal{Z} \rightarrow \tilde{\Delta}\left(\left(X_{w}\right)_{j}\right)$ is affine.

Proof. Since $\tilde{\Delta}\left(\left(X_{w}\right)_{j}\right)$ is a closed subscheme of $\left(X_{w}^{2}\right)_{\mathbb{P}}$ it suffices to show that the map $m: \bar{\Gamma} \times\left(X_{w}^{u, v}\right)_{\mathbb{P}} \rightarrow\left(X_{w}^{2}\right)_{\mathbb{P}}$ is an affine morphism. Now, if $f: X \rightarrow Y$ is an affine morphism and $Z \subset X$ is a closed subscheme, then $\left.f\right|_{Z}: Z \rightarrow Y$ is clearly an affine morphism. Thus, it suffices to show that $\bar{\Gamma} \times\left(X_{w}^{2}\right)_{\mathbb{P}} \rightarrow\left(X_{w}^{2}\right)_{\mathbb{P}}$ is an affine morphism. Further, if $X$ and $Y$ are total spaces of principal $T$-bundles and $f: X \rightarrow Y$ is a $T$-equivariant map, then $f$ is affine iff $\bar{f}: X / T \rightarrow Y / T$ is affine. Thus, it suffices to show that

$$
\hat{\mu}: \bar{\Gamma} \times\left(E(T)_{\mathbb{P}} \times X_{w}^{2}\right) \rightarrow E(T)_{\mathbb{P}} \times X_{w}^{2}
$$

is affine. Recall that $\bar{\Gamma}=\bar{\Gamma}_{0} \rtimes G L(N+1)^{r}$ and $\hat{\mu}$ is given by $\hat{\mu}((\sigma, g),(e, x))=$ $(g e, \sigma(g e) \cdot x)$, where $\sigma \in \bar{\Gamma}_{0}, g \in G L(N+1)^{r}, e \in E(T)_{\mathbb{P}}, x \in X_{w}^{2}$. Write $\hat{\mu}$ as a composite $\hat{\mu}=\mu_{3} \circ \mu_{2} \circ \mu_{1}$, where

$\mu_{1}: \bar{\Gamma}_{0} \times G L(N+1)^{r} \times E(T)_{\mathbb{P}} \times X_{w}^{2} \rightarrow \bar{\Gamma}_{0} \times E(T)_{\mathbb{P}} \times X_{w}^{2}, \quad(\sigma, g, e, x) \mapsto(\sigma, g \cdot e, x)$,

$$
\mu_{2}: \bar{\Gamma}_{0} \times E(T)_{\mathbb{P}} \times X_{w}^{2} \rightarrow \bar{B}^{2} \times E(T)_{\mathbb{P}} \times X_{w}^{2}, \quad(\sigma, e, x) \mapsto(\sigma(e), e, x),
$$$$
\mu_{3}: \bar{B}^{2} \times E(T)_{\mathbb{P}} \times X_{w}^{2} \rightarrow E(T)_{\mathbb{P}} \times X_{w}^{2}, \quad\left(\left(b_{1}, b_{2}\right), e,(x, y)\right) \mapsto\left(e, b_{1} x, b_{2} y\right) .
$$

As the composition of two affine morphisms is affine, it suffices to prove that $\mu_{1}, \mu_{2}, \mu_{3}$ are affine. Moreover, if $f: X \rightarrow Y$ is affine, then $f \times \operatorname{Id}_{Z}: X \times Z \rightarrow Y \times Z$ is affine for any scheme $Z$. Hence, it suffices to prove that the following maps $\hat{\mu}_{1}, \hat{\mu}_{2}$, and $\hat{\mu}_{3}$ are affine:

$$
\begin{aligned}
& \hat{\mu}_{1}: G L(N+1)^{r} \times E(T)_{\mathbb{P}} \rightarrow E(T)_{\mathbb{P}},(g, e) \mapsto g \cdot e, \\
& \hat{\mu}_{2}: \bar{\Gamma}_{0} \times E(T)_{\mathbb{P}} \rightarrow \bar{B}^{2} \times E(T)_{\mathbb{P}},(\sigma, e) \mapsto(\sigma(e), e), \\
& \hat{\mu}_{3}: \bar{B}^{2} \times X_{w}^{2} \rightarrow X_{w}^{2},\left(\left(b_{1}, b_{2}\right),(x, y)\right) \mapsto\left(b_{1} x, b_{2} y\right) .
\end{aligned}
$$

(1) $\hat{\mu}_{1}$ is affine: Since $E(T)_{\mathbb{P}}=\left(\mathbb{C}^{N+1} \backslash\{0\}\right)^{r}$, it suffices to prove that $\theta$ : $G L(N+1) \times\left(\mathbb{C}^{N+1} \backslash\{0\}\right) \rightarrow \mathbb{C}^{N+1} \backslash\{0\},(g, v) \mapsto g \cdot v$ is affine. Now, consider the map $\bar{\theta}: G L(N+1) \times \mathbb{C}^{N+1} \rightarrow \mathbb{C}^{N+1},(g, v) \mapsto g \cdot v$. Since both the domain and codomain are affine, $\bar{\theta}$ is an affine morphism. Moreover, $\theta=\left.\bar{\theta}\right|_{\bar{\theta}^{-1}\left(\mathbb{C}^{N+1} \backslash\{0\}\right)}$. Thus, $\theta$ is affine.

(2) $\hat{\mu}_{2}$ is affine: Take an affine open subset $\mathcal{U} \subset E(T)_{\mathbb{P}}$. Then, $\bar{B}^{2} \times \mathcal{U}$ is an affine open subset in $\bar{B}^{2} \times E(T)_{\mathbb{P}}$. Now, $\hat{\mu}_{2}^{-1}\left(\bar{B}^{2} \times \mathcal{U}\right)=\bar{\Gamma}_{0} \times \mathcal{U}$. Since $\bar{\Gamma}_{0}$ is affine, so is $\bar{\Gamma}_{0} \times \mathcal{U}$. Thus, $\hat{\mu}_{2}$ is affine. 
(3) $\hat{\mu}_{3}$ is affine: It suffices to prove that $\delta: \bar{B} \times X_{w} \rightarrow X_{w},(b, x) \mapsto b x$ is affine. Take an affine open subset $V \subset X_{w}$. Then, $\delta^{-1}(V)=\bigcup_{b \in \bar{B}}\left(b, b^{-1} V\right)$. Consider the scheme isomorphism $i: \bar{B} \times X_{w} \mapsto \bar{B} \times X_{w},(b, x) \mapsto(b, b \cdot x)$. Then, $i\left(\delta^{-1}(V)\right)=\bar{B} \times V$. But, since $\bar{B} \times V$ is affine, so is $\delta^{-1}(V)$. Thus, $\delta$ is an affine morphism and hence so is $\hat{\mu}_{3}$.

Let $\pi: \mathcal{Z} \rightarrow \bar{\Gamma}$ denote the projection onto the first factor and $\pi_{1}: \partial \mathcal{Z} \rightarrow \bar{\Gamma}$ denote the restriction of $\pi$ to $\partial \mathcal{Z}$. We define the fibers

$$
N_{\gamma}:=\pi^{-1}(\gamma) \simeq \gamma\left(\left(X_{w}^{u, v}\right)_{\mathbb{P}}\right) \cap \tilde{\Delta}\left(\left(X_{w}\right)_{j}\right)
$$

and

$$
M_{\gamma}:=\pi_{1}^{-1}(\gamma) \simeq \gamma\left(\left(X_{w}^{u, v}\right)_{\mathbb{P}}\right) \cap \tilde{\Delta}\left(\partial\left(\left(X_{w}\right)_{j}\right)\right) .
$$

Corollary 7.10. Assume that $c_{u, v}^{w}(j) \neq 0$, where $c_{u, v}^{w}(j)$ are defined by (4). Then, for general $\gamma \in \bar{\Gamma}$, we have that $N_{\gamma}$ (defined by (7)) is CM of pure dimension. In fact, for $\gamma \in \bar{\Gamma}$ such that

$$
\operatorname{dim} N_{\gamma}=\operatorname{dim} \mathcal{Z}-\operatorname{dim} \bar{\Gamma}=|j|+\ell(w)-\ell(u)-\ell(v),
$$

$N_{\gamma}$ is CM, and this condition is satisfied for general $\gamma$.

Similarly, if $|j|+\ell(w)-\ell(u)-\ell(v)>0$, for general $\gamma \in \bar{\Gamma}$, we have that $M_{\gamma}$ (defined by 8) is CM of pure codimension 1 in $N_{\gamma}$. If $|j|+\ell(w)-\ell(u)-\ell(v)=0$, for general $\gamma \in \bar{\Gamma}$, we have that $M_{\gamma}$ is empty.

Proof. First we show that $\pi$ is surjective when $c_{u, v}^{w}(j) \neq 0$. From the definition of $\pi$ we have that

$$
\operatorname{Im} \pi=\left\{\gamma \in \bar{\Gamma}: \gamma\left(\left(X_{w}^{u, v}\right)_{\mathbb{P}}\right) \cap \tilde{\Delta}\left(\left(X_{w}\right)_{j}\right) \neq \emptyset\right\} .
$$

Since $\bar{\Gamma}$ is connected, Lemma 4.10 along with the assumption $c_{u, v}^{w}(j) \neq 0$ gives that $\gamma\left(\left(X^{u, v}\right)_{\mathbb{P}}\right) \cap \tilde{\Delta}\left(\left(X_{w}\right)_{j}\right) \neq \emptyset$ for any $\gamma \in \bar{\Gamma}$. Since $\gamma\left(\left(X^{u, v}\right)_{\mathbb{P}}\right) \cap \tilde{\Delta}\left(\left(X_{w}\right)_{j}\right)=$ $\gamma\left(\left(X_{w}^{u, v}\right)_{\mathbb{P}}\right) \cap \tilde{\Delta}\left(\left(X_{w}\right)_{j}\right)$, we get that $\pi$ is surjective.

Now, since $\mathcal{Z}$ is $\mathrm{CM}$, applying Lemma 7.4 to $\pi$ gives that if

$$
\operatorname{codim}_{\mathcal{Z}}\left(N_{\gamma}\right)=\operatorname{dim} \bar{\Gamma}
$$

then $N_{\gamma}$ is CM. By [S, Chapter I, $\S 6.3$, Theorem 1.25], this condition holds for $\gamma$ in a dense open subset of $\bar{\Gamma}$. Thus, $N_{\gamma}$ is CM for $\gamma$ satisfying $\operatorname{dim} N_{\gamma}=\operatorname{dim} \mathcal{Z}-\operatorname{dim} \bar{\Gamma}$, and this condition is satisfied for general $\gamma$.

Next, we show that if $|j|+\ell(w)-\ell(u)-\ell(v)>0$, then $\pi_{1}$ is surjective. First note that since $\pi_{1}$ is projective, if it is not surjective, its image is a proper closed subset of $\bar{\Gamma}$. Thus, for general $\gamma \in \bar{\Gamma}, M_{\gamma}=\emptyset$, i.e., $N_{\gamma} \subset \mathcal{Z} \backslash \partial \mathcal{Z}$. As $\mu$ is an affine morphism by Lemma 7.9, $\mathcal{Z} \backslash \partial \mathcal{Z}$ is affine. But, $N_{\gamma}$ is projective of positive dimension (since $|j|+\ell(w)-\ell(u)-\ell(v)>0$ ) which gives a contradiction.

In particular, there is at least one irreducible component of $\partial \mathcal{Z}$ on which $\pi_{1}$ is surjective. The other irreducible components are mapped to closed subsets of $\bar{\Gamma}$. Let $\mathcal{U}$ be the complement of the union of the images of the irreducible components on which $\pi_{1}$ is not surjective. Then, $\mathcal{U}$ is open, since there are only finitely many irreducible components. Applying [S, Chapter I, §6.3, Theorem 1.25] to $\pi_{1}$ on each irreducible component of $\partial \mathcal{Z}$ which surjects onto $\bar{\Gamma}$ and then intersecting with $\mathcal{U}$ gives that, for general $\gamma \in \bar{\Gamma}$,

$$
\operatorname{codim}_{\partial \mathcal{Z}}\left(M_{\gamma}\right)=\operatorname{dim} \bar{\Gamma} .
$$


Thus, by Lemma 7.4, $M_{\gamma}$ is CM. Moreover, (9) and (10) together imply that $M_{\gamma}$ is pure of codimension 1 in $N_{\gamma}$.

In the case where $|j|+\ell(w)-\ell(u)-\ell(v)=0$, we have $\operatorname{dim} \mathcal{Z}=\operatorname{dim} \bar{\Gamma}$, so that $\operatorname{dim} \partial \mathcal{Z}<\operatorname{dim} \bar{\Gamma}$. Thus, $\operatorname{Im} \pi_{1}$ is a proper closed subset of $\bar{\Gamma}$, and hence $M_{\gamma}$ is empty for general $\gamma$.

Corollary 7.11. Assume that $c_{u, v}^{w}(j) \neq 0$. Then, for general $\gamma \in \bar{\Gamma}$, we have

$$
\mathscr{E} x t_{\mathcal{O}_{N_{\gamma}}}^{i}\left(\mathcal{O}_{N_{\gamma}}\left(-M_{\gamma}\right), \omega_{N_{\gamma}}\right)=0, \forall i>0 .
$$

Proof. By [I, Proposition 11.33 and Corollary 11.43] we have

$$
\mathscr{E} x t_{\mathcal{O}_{N_{\gamma}}}^{i}\left(\mathcal{O}_{M_{\gamma}}, \omega_{N_{\gamma}}\right)=0 \text {, unless } i=1
$$

and, of course,

$$
\mathscr{E} x t_{\mathcal{O}_{N_{\gamma}}}^{i}\left(\mathcal{O}_{N_{\gamma}}, \omega_{N_{\gamma}}\right)=0, \forall i>0
$$

Hence, the desired result follows from the long exact $\mathscr{E} x t$ sequence associated to the short exact sequence

$$
0 \rightarrow \mathcal{O}_{N_{\gamma}}\left(-M_{\gamma}\right) \rightarrow \mathcal{O}_{N_{\gamma}} \rightarrow \mathcal{O}_{M_{\gamma}} \rightarrow 0
$$

\section{Application of Kawamata-Viehweg Vanishing}

In this section we assume that $c_{u, v}^{w}(j) \neq 0$.

Let $f: X \rightarrow Y$ be a proper morphism between schemes, with $X$ irreducible, and let $\mathcal{M}$ be a line bundle on $X$. Then, $\mathcal{M}$ is said to be $f$-nef if it has non-negative intersection with every curve contained in a fiber of $f$. The line bundle $\mathcal{M}$ is said to be $f$-big if rank $f_{*}\left(\mathcal{M}^{k}\right)>c \cdot k^{n}$ for some $c>0$ and $k \gg 1$, where $n$ is the dimension of a general fiber of $f$. A Weil divisor $D \subset X$ has normal crossings if all of its irreducible components intersect transversely. It is easy to see that $\partial Z_{w}$ and hence $\partial\left(\left(Z_{w}\right)_{j}\right)$ are normal crossings divisors in $Z_{w}$ and $\left(Z_{w}\right)_{j}$, respectively.

We will need the following relative Kawamata-Viehweg vanishing theorem from [AGM, Theorem 2.4], which was originally extracted from [EV, Corollary 6.11].

Theorem 8.1. Let $f: \widetilde{Z} \rightarrow Z$ be a proper surjective morphism of varieties, with $\widetilde{Z}$ non-singular. Let $\mathcal{M}$ be a line bundle on $\widetilde{Z}$ such that $\mathcal{M}^{\bar{N}}(-D)$ is $f$-nef and $f$-big for a normal crossing divisor $D=\sum_{j=1}^{r} a_{j} D_{j}$, where $0<a_{j}<\bar{N}$ for all $j$. Then,

$$
R^{i} f_{*}\left(\mathcal{M} \otimes \omega_{\widetilde{Z}}\right)=0, \forall i>0 .
$$

Definition 8.2. We define the sheaf

$$
\omega_{\mathcal{Z}}(\partial \mathcal{Z}):=\mathscr{H}_{o m_{\mathcal{O}_{\mathcal{Z}}}}\left(\mathcal{O}_{\mathcal{Z}}(-\partial \mathcal{Z}), \omega_{\mathcal{Z}}\right) .
$$

Theorem 8.3. We have $f_{*} \omega_{\widetilde{\mathcal{Z}}}(\partial \widetilde{\mathcal{Z}})=\omega_{\mathcal{Z}}(\partial \mathcal{Z})$, where $\widetilde{\mathcal{Z}}, \mathcal{Z}, \partial \widetilde{\mathcal{Z}}, \partial \mathcal{Z}$, and the morphism $f: \widetilde{\mathcal{Z}} \rightarrow \mathcal{Z}$ are defined after Lemma $\mathbf{7 . 2}$. (Observe that since $\mathcal{Z}$ is $\mathrm{CM}$, the dualizing sheaf $\omega_{\mathcal{Z}}$ makes sense.)

Proof. First, we claim

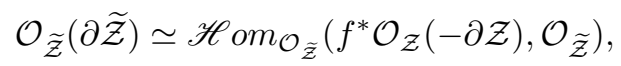

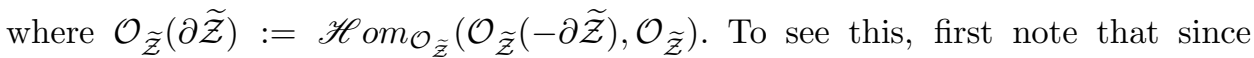
$f^{-1}(\partial \mathcal{Z})=\partial \widetilde{\mathcal{Z}}$ is the scheme-theoretic inverse image (cf. $\S 6$ ), the natural morphism $f^{*} \mathcal{O}_{\mathcal{Z}}(-\partial \mathcal{Z}) \rightarrow \mathcal{O}_{\widetilde{\mathcal{Z}}}(-\partial \widetilde{\mathcal{Z}})$ is surjective (cf., e.g., [Stacks, Tag 01HJ] Lemma 25.4.7]). 
As $f$ is a desingularization (cf. Proposition 7.5), the kernel of this morphism is supported on a proper closed subset of $\widetilde{\mathcal{Z}}$ and hence is a torsion sheaf. This implies that the dual map $\mathcal{O}_{\widetilde{\mathcal{Z}}}(\partial \widetilde{\mathcal{Z}}) \rightarrow \mathscr{H}^{\circ} m_{\mathcal{O}_{\tilde{\mathcal{Z}}}}\left(f^{*} \mathcal{O}_{\mathcal{Z}}(-\partial \mathcal{Z}), \mathcal{O}_{\widetilde{\mathcal{Z}}}\right)$ is an isomorphism, proving (11).

To complete the proof of the theorem, we compute:

$$
\begin{aligned}
& f_{*}\left(\omega_{\widetilde{\mathcal{Z}}} \otimes \mathcal{O}_{\widetilde{\mathcal{Z}}}(\partial \widetilde{\mathcal{Z}})\right)=f_{*}\left(\omega_{\widetilde{\mathcal{Z}}} \otimes \mathscr{H}_{o m_{\mathcal{O}_{\tilde{\mathcal{Z}}}}}\left(f^{*} \mathcal{O}_{\mathcal{Z}}(-\partial \mathcal{Z}), \mathcal{O}_{\widetilde{\mathcal{Z}}}\right)\right), \text { by (11) } \\
& =f_{*} \mathscr{H}_{o m_{\mathcal{O}_{\tilde{\mathcal{Z}}}}}\left(f^{*} \mathcal{O}_{\mathcal{Z}}(-\partial \mathcal{Z}), \omega_{\widetilde{\mathcal{Z}}}\right) \\
& =\mathscr{H}_{o m_{\mathcal{Z}}}\left(\mathcal{O}_{\mathcal{Z}}(-\partial \mathcal{Z}), f_{*} \omega_{\widetilde{\mathcal{Z}}}\right) \text {, by adjunction (cf. [H, Chapter II, §5]) } \\
& =\mathscr{H}_{o m_{\mathcal{O}_{\mathcal{Z}}}}\left(\mathcal{O}_{\mathcal{Z}}(-\partial \mathcal{Z}), \omega_{\mathcal{Z}}\right) \text {, by Proposition } 7.7 \text { and }[\mathrm{KM} \text {, Theorem 5.10] } \\
& =\omega_{\mathcal{Z}}(\partial \mathcal{Z})
\end{aligned}
$$

Lemma 8.4. The homogeneous line bundle $\left.\mathcal{L}(\rho)\right|_{X_{w}}$ (cf. §2) has a section with zero set exactly equal to $\partial X_{w}$.

Proof. Consider the Borel-Weil homomorphism (cf. [Ku2, §8.1.21]) $\chi_{w}: L(\rho)^{*} \rightarrow$ $H^{0}\left(X_{w}, \mathcal{L}(\rho)\right)$ which is given by $\chi_{w}(f)(g B)=\left[g, f\left(g e_{\rho}\right)\right]$, where $e_{\rho}$ is the highest weight vector of the irreducible highest weight $G^{\text {min }}$-module $L(\rho)$ with highest weight $\rho$.

Consider the section $\chi_{w}\left(e_{w \rho}^{*}\right)$, where $e_{w \rho}$ is the weight vector of $L(\rho)$ with weight $w \rho$ and $e_{w \rho}^{*} \in L(\rho)^{*}$ is the linear form which takes the value 1 on $e_{w \rho}$ and 0 on any weight vector of $L(\rho)$ of weight different from $w \rho$. Let $w^{\prime} \leq w$ and $b \in B$. We have

$$
\chi_{w}\left(e_{w \rho}^{*}\right)\left(b w^{\prime} B\right)=\left[b w^{\prime}, e_{w \rho}^{*}\left(b w^{\prime} e_{\rho}\right)\right] .
$$

Now, for $w^{\prime}<w$, we have $w^{\prime} \rho>w \rho$ by [Ku2, Lemma 8.3.3]. Thus, $e_{w \rho}^{*}\left(b w^{\prime} e_{\rho}\right)=0$ for any $b \in B$ and $w^{\prime}<w$. For $w^{\prime}=w$, we have $e_{w \rho}^{*}\left(b w e_{\rho}\right) \neq 0$ for any $b \in B$. Hence, $\chi_{w}\left(e_{w \rho}^{*}\right)$ has zero set precisely equal to $\partial X_{w}$.

Lemma 8.5. There is an ample line bundle $\mathcal{L}$ on $\left(X_{w}\right)_{j}$ with a section with zero set exactly equal to $\partial\left(\left(X_{w}\right)_{j}\right)$.

Proof. By the previous lemma, the ample line bundle $\left.\mathcal{L}(\rho)\right|_{X_{w}}$ has a section with zero set exactly equal to $\partial X_{w}$. The $T$-equivariant line bundle $\left.\left(e^{w \rho} \mathcal{L}(\rho)\right)\right|_{X_{w}}$ gives rise to the line bundle

$$
\left(\left.e^{w \rho} \mathcal{L}(\rho)\right|_{X_{w}}\right)_{j}:=E(T)_{\mathbb{P}_{j}} \times^{T}\left(\left.\left(e^{w \rho} \mathcal{L}(\rho)\right)\right|_{X_{w}}\right)
$$

on $\left(X_{w}\right)_{j}$. Then, the section $\theta$ defined by $[e, x] \mapsto\left[e, 1_{w \rho} \otimes \chi_{w}\left(e_{w \rho}^{*}\right) x\right]$ for $e \in E(T)_{\mathbb{P}_{j}}$ and $x \in X_{w}$ has zero set exactly equal to $\left(\partial X_{w}\right)_{j}$, where $1_{w \rho}$ is a non-zero element of the line bundle $e^{w \rho}$ (cf. $\S 2$ ).

Now, let $\mathcal{H}$ be an ample line bundle on $\mathbb{P}_{j}$ with a section $\sigma$ with zero set exactly $\partial \mathbb{P}_{j}$ and consider the bundle

$$
\mathcal{L}:=\left(\left.e^{w \rho} \mathcal{L}(\rho)\right|_{X_{w}}\right)_{j} \otimes p^{*}\left(\mathcal{H}^{\bar{N}}\right),
$$


where $p:\left(X_{w}\right)_{j} \rightarrow \mathbb{P}_{j}$ is the projection. Let $\hat{\sigma}$ represent the pullback of $\sigma$. Then, the section $\theta \otimes(\hat{\sigma})^{\bar{N}}$ has zero set exactly equal to $\partial\left(\left(X_{w}\right)_{j}\right)$. Furthermore, by [KM, Proposition 1.45], if $\bar{N}$ is large enough, $\mathcal{L}$ is ample.

Lemma 8.6. Let $f: \widetilde{A} \rightarrow A$ be a proper birational map between normal, irreducible varieties and let $\pi: A \rightarrow B$ be a surjective proper morphism to a variety $B$. Let $\tilde{\mathcal{L}}$ be a $\pi$-big line bundle on $A$. Then, the pullback line bundle $f^{*} \tilde{\mathcal{L}}$ is $\tilde{\pi}$-big, where $\tilde{\pi}=\pi \circ f: \widetilde{A} \rightarrow B$.

Proof. It suffices to show that $\operatorname{rank} \tilde{\pi}_{*}\left(f^{*} \tilde{\mathcal{L}}^{k}\right)>c \cdot k^{n}$ for some $c>0$ and $k \gg 1$, where $n$ is the dimension of a general fiber of $\tilde{\pi}$.

By [S. Chapter $1, \S 6.3$, Theorem 1.25], the dimension of a general fiber of $\pi$ and the dimension of a general fiber of $\tilde{\pi}$ are both equal to $\operatorname{dim} A-\operatorname{dim} B$.

Now, $\tilde{\pi}_{*}\left(f^{*} \tilde{\mathcal{L}}^{k}\right)=\pi_{*} f_{*}\left(f^{*} \tilde{\mathcal{L}}^{k}\right) \simeq \pi_{*}\left(\tilde{\mathcal{L}}^{k}\right)$ by the projection formula, since $A$ is normal. Hence, rank $\tilde{\pi}_{*}\left(f^{*} \tilde{\mathcal{L}}^{k}\right)>c \cdot k^{n}$ for some $c>0$ and $k \gg 1$, where $n$ is the dimension of a general fiber of $\pi$, since $\tilde{\mathcal{L}}$ is $\pi$-big. This proves the lemma.

Theorem 8.7. Assume that $c_{u, v}^{w}(j) \neq 0$. Then, for all $i>0$, we have $R^{i} \tilde{\pi}_{*} \omega_{\widetilde{\mathcal{Z}}}(\partial \widetilde{\mathcal{Z}})=$ 0 and $R^{i} f_{*} \omega_{\widetilde{\mathcal{Z}}}(\partial \widetilde{\mathcal{Z}})=0$, where $\tilde{\pi}: \widetilde{\mathcal{Z}} \rightarrow \bar{\Gamma}$ is the projection onto the first factor and $f: \widetilde{\mathcal{Z}} \rightarrow \mathcal{Z}$ is defined after Lemma 7.2 .

Proof. Since $f$ is proper and birational by Proposition 7.5, it is surjective. By the proof of Corollary 7.10 , the projection $\pi: \mathcal{Z} \rightarrow \bar{\Gamma}$ is also surjective. Thus, $\tilde{\pi}=\pi \circ f$ is also surjective. Moreover, $\tilde{\pi}$ is proper since it is the restriction of the projection $\bar{\Gamma} \times\left(Z_{w}^{u, v}\right)_{\mathbb{P}} \rightarrow \bar{\Gamma}$ to the closed subset $\widetilde{\mathcal{Z}}$, where $\left(Z_{w}^{u, v}\right)_{\mathbb{P}}$ is projective. Let $\mathcal{M}$ denote the line bundle $\mathcal{M}:=\mathcal{O}_{\widetilde{\mathcal{Z}}}(\partial \widetilde{\mathcal{Z}})$. (Observe that $\partial \widetilde{\mathcal{Z}}$ is a divisor in non-singular $\widetilde{\mathcal{Z}}$.) By Theorem 8.1, it suffices to find a normal crossings divisor $D \subset \widetilde{\mathcal{Z}}$ such that the line bundle $\mathcal{M}^{\bar{N}}(-D)$ is $\tilde{\pi}$-nef, $f$-nef, $\tilde{\pi}$-big, and $f$-big and $D=\sum_{j=1}^{r} a_{j} D_{j}$, with $0<a_{j}<\bar{N}$.

By Lemma 8.5 we may choose an ample line bundle $\mathcal{L}$ on $\tilde{\Delta}\left(\left(X_{w}\right)_{j}\right)$ with a section with zero set exactly equal to $\tilde{\Delta}\left(\partial\left(\left(X_{w}\right)_{j}\right)\right)$. Let $\varphi: \tilde{\Delta}\left(\left(Z_{w}\right)_{j}\right) \rightarrow \tilde{\Delta}\left(\left(X_{w}\right)_{j}\right)$ denote the desingularization. Since $\partial\left(\left(Z_{w}\right)_{j}\right)$ is a normal crossings divisor in $\left(Z_{w}\right)_{j}$, it follows that $\partial \widetilde{\mathcal{Z}}=\left(\bar{\Gamma} \times\left(Z_{w}^{u, v}\right)_{\mathbb{P}}\right) \times_{\left(Z_{w}^{2}\right)_{\mathbb{P}}} \widetilde{\Delta}\left(\partial\left(\left(Z_{w}\right)_{j}\right)\right)$ is a normal crossings divisor in $\widetilde{\mathcal{Z}}$. Write

$$
\partial \widetilde{\mathcal{Z}}=D_{1}+\cdots+D_{\ell}
$$

where $D_{i}$ are the irreducible components. Since $\tilde{\mu}^{*} \varphi^{*} \mathcal{L}$ has a section with zero set precisely equal to $\partial \widetilde{\mathcal{Z}}$, it follows that

$$
\tilde{\mu}^{*} \varphi^{*} \mathcal{L}=\mathcal{O}_{\widetilde{\mathcal{Z}}}\left(b_{1} D_{1}+\cdots+b_{\ell} D_{\ell}\right)
$$

for some positive integers $b_{1}, \ldots, b_{\ell}$.

Let $D$ be the divisor $D:=a_{1} D_{1}+\cdots+a_{\ell} D_{\ell}$ where $a_{i}:=\bar{N}-b_{i}$ for some integer $\bar{N}$ greater than all the $b_{i}$ 's. Since $\partial \widetilde{\mathcal{Z}}$ has normal crossings, so does $D$. Then,

$$
\mathcal{M}^{\bar{N}}(-D)=\mathcal{O}_{\widetilde{\mathcal{Z}}}\left(b_{1} D_{1}+\cdots+b_{\ell} D_{\ell}\right)=\tilde{\mu}^{*} \varphi^{*} \mathcal{L}
$$

Since the fibers of $\tilde{\pi}$ are projective schemes and $\mathcal{L}$ is an ample line bundle on $\tilde{\Delta}\left(\left(X_{w}\right)_{j}\right)$, the pullback $\tilde{\mu}^{*} \varphi^{*} \mathcal{L}$ restricted to the fibers of $\tilde{\pi}$ is nef, since the pullback of any ample line bundle under a morphism between projective varieties is nef 
(cf. D. Theorem 1.26, $\S 1.9$ and $\S 1.29]$ ). Thus, $\mathcal{M}^{\bar{N}}(-D)$ is $\tilde{\pi}$-nef. Since the fibers of $f$ are contained in the fibers of $\tilde{\pi}, \mathcal{M}^{\bar{N}}(-D)$ is also $f$-nef.

Now, $\mathcal{M}^{\bar{N}}(-D)$ is $f$-big since $f$ is birational by Proposition 7.5. It remains to show $\tilde{\pi}$-bigness. Clearly, $\mu: \mathcal{Z} \rightarrow \tilde{\Delta}\left(\left(X_{w}\right)_{j}\right)$ is a closed embedding restricted to any fiber of the morphism $\pi: \mathcal{Z} \rightarrow \bar{\Gamma}$. Hence, the ample line bundle $\mathcal{L}$ on $\tilde{\Delta}\left(\left(X_{w}\right)_{j}\right)$ pulls back to a $\pi$-big line bundle $\mu^{*} \mathcal{L}$ on $\mathcal{Z}$. Now, $\tilde{\pi}$-bigness of $\mathcal{M}^{\bar{N}}(-D)=\tilde{\mu}^{*} \varphi^{*} \mathcal{L}=$ $f^{*} \mu^{*} \mathcal{L}$ follows from Lemma 8.6 and Proposition 7.5 .

Theorems 8.3 and 8.7 together with the Grothendieck spectral sequence give

Corollary 8.8. For all $i>0$ we have $R^{i} \pi_{*} \omega_{\mathcal{Z}}(\partial \mathcal{Z})=0$.

\section{Proof of part b) of Theorem 5.1}

Recall the definitions of $N_{\gamma}$ and $M_{\gamma}$ from equations (7) and (8).

We also define

and

$$
M_{\gamma}^{1}:=\gamma\left(\left(X_{w}^{u, v}\right)_{\mathbb{P}}\right) \cap \tilde{\Delta}\left(\left(X_{w}\right)_{\partial \mathbb{P}_{j}}\right)
$$

so that

$$
M_{\gamma}^{2}:=\gamma\left(\left(X_{w}^{u, v}\right)_{\mathbb{P}}\right) \cap \tilde{\Delta}\left(\left(\partial X_{w}\right)_{j}\right),
$$

$$
M_{\gamma}=M_{\gamma}^{1} \cup M_{\gamma}^{2} .
$$

Lemma 9.1. For general $\gamma \in \bar{\Gamma}$, the sheaf

$$
\gamma_{*} \mathcal{O}_{\left(X^{u} \times X^{v}\right)_{\mathbb{P}}} \otimes_{\mathcal{O}_{\bar{Y}_{\mathbb{P}}}}\left(\hat{p}^{*}\left(\mathcal{O}_{\mathbb{P}_{j}}\left(-\partial \mathbb{P}_{j}\right)\right) \otimes_{\mathcal{O}_{Y_{\mathbb{P}}}} \tilde{\Delta}_{*}\left(\left(\xi_{w}\right)_{\mathbb{P}}\right)\right)
$$

is supported on $N_{\gamma}$ and is equal to the sheaf $\mathcal{O}_{N_{\gamma}}\left(-M_{\gamma}\right)$, where $\hat{p}: Y_{\mathbb{P}} \rightarrow \mathbb{P}$ is the projection.

Proof. Since $\hat{p}: Y_{\mathbb{P}} \rightarrow \mathbb{P}$ is flat, we have a short exact sequence

$$
0 \rightarrow \hat{p}^{*}\left(\mathcal{O}_{\mathbb{P}_{j}}\left(-\partial \mathbb{P}_{j}\right)\right) \rightarrow \hat{p}^{*} \mathcal{O}_{\mathbb{P}_{j}} \rightarrow \hat{p}^{*} \mathcal{O}_{\partial \mathbb{P}_{j}} \rightarrow 0 .
$$

By Lemma 4.9 tensoring with $\tilde{\Delta}_{*}\left(\left(\xi_{w}\right)_{\mathbb{P}}\right)$ over $\mathcal{O}_{Y_{\mathbb{P}}}$ preserves exactness of the above sequence, so we have an exact sequence

$$
\begin{aligned}
0 \rightarrow \hat{p}^{*}\left(\mathcal{O}_{\mathbb{P}_{j}}\left(-\partial \mathbb{P}_{j}\right)\right) \otimes_{\mathcal{O}_{Y_{\mathbb{P}}}} \tilde{\Delta}_{*}\left(\left(\xi_{w}\right) \mathbb{P}\right) & \rightarrow \hat{p}^{*} \mathcal{O}_{\mathbb{P}_{j}} \otimes_{\mathcal{O}_{Y_{\mathbb{P}}}} \tilde{\Delta}_{*}\left(\left(\xi_{w}\right)_{\mathbb{P}}\right) \\
& \rightarrow \hat{p}^{*} \mathcal{O}_{\partial \mathbb{P}_{j}} \otimes_{\mathcal{O}_{Y_{\mathbb{P}}}} \tilde{\Delta}_{*}\left(\left(\xi_{w}\right)_{\mathbb{P}}\right) \rightarrow 0 .
\end{aligned}
$$

By Theorem 6.2, for general $\gamma \in \bar{\Gamma}$, tensoring the above sequence with $\gamma_{*} \mathcal{O}_{\left(X^{u} \times X^{v}\right)_{\mathbb{P}}}$ over $\mathcal{O}_{\bar{Y}_{\mathbb{P}}}$ preserves exactness, so we have, for general $\gamma \in \bar{\Gamma}$, an exact sequence

$$
\begin{aligned}
0 & \rightarrow \gamma_{*} \mathcal{O}_{\left(X^{u} \times X^{v}\right)_{\mathbb{P}}} \otimes_{\mathcal{O}_{\bar{Y}_{\mathbb{P}}}}\left(\hat{p}^{*}\left(\mathcal{O}_{\mathbb{P}_{j}}\left(-\partial \mathbb{P}_{j}\right)\right) \otimes_{\mathcal{O}_{Y_{\mathbb{P}}}} \tilde{\Delta}_{*}\left(\left(\xi_{w}\right)_{\mathbb{P}}\right)\right) \\
& \rightarrow \gamma_{*} \mathcal{O}_{\left(X^{u} \times X^{v}\right)_{\mathbb{P}}} \otimes_{\mathcal{O}_{\bar{Y}}}\left(\hat{p}^{*} \mathcal{O}_{\mathbb{P}_{j}} \otimes_{\mathcal{O}_{Y_{\mathbb{P}}}} \tilde{\Delta}_{*}\left(\left(\xi_{w}\right)_{\mathbb{P}}\right)\right) \\
& \rightarrow \gamma_{*} \mathcal{O}_{\left(X^{u} \times X^{v}\right)_{\mathbb{P}}} \otimes_{\mathcal{O}_{\bar{Y}_{\mathbb{P}}}}\left(\hat{p}^{*} \mathcal{O}_{\partial \mathbb{P}_{j}} \otimes_{\mathcal{O}_{Y_{\mathbb{P}}}} \tilde{\Delta}_{*}\left(\left(\xi_{w}\right)_{\mathbb{P}}\right)\right) \rightarrow 0 .
\end{aligned}
$$

Next, consider the exact sequence

$$
0 \rightarrow \mathcal{O}_{N_{\gamma}}\left(-M_{\gamma}\right) \rightarrow \mathcal{O}_{N_{\gamma}}\left(-M_{\gamma}^{2}\right) \rightarrow \mathcal{O}_{M_{\gamma}^{1}}\left(-M_{\gamma}^{1} \cap M_{\gamma}^{2}\right) \rightarrow 0 .
$$

Comparing (12) with (13) we see that it is sufficient to show that

$$
\gamma_{*} \mathcal{O}_{\left(X^{u} \times X^{v}\right)_{\mathbb{P}}} \otimes_{\mathcal{O}_{\bar{Y}_{\mathbb{P}}}}\left(\hat{p}^{*} \mathcal{O}_{\mathbb{P}_{j}} \otimes_{\mathcal{O}_{Y_{\mathbb{P}}}} \tilde{\Delta}_{*}\left(\left(\xi_{w}\right)_{\mathbb{P}}\right)\right)=\mathcal{O}_{N_{\gamma}}\left(-M_{\gamma}^{2}\right)
$$


and

$$
\gamma_{*} \mathcal{O}_{\left(X^{u} \times X^{v}\right)_{\mathbb{P}}} \otimes_{\mathcal{O}_{\bar{Y}_{\mathbb{P}}}}\left(\hat{p}^{*} \mathcal{O}_{\partial \mathbb{P}_{j}} \otimes_{\mathcal{O}_{Y_{\mathbb{P}}}} \tilde{\Delta}_{*}\left(\left(\xi_{w}\right)_{\mathbb{P}}\right)\right)=\mathcal{O}_{M_{\gamma}^{1}}\left(-M_{\gamma}^{1} \cap M_{\gamma}^{2}\right)
$$

To prove (14) and (15) consider the short exact sequence

$$
0 \rightarrow \tilde{\Delta}_{*}\left(\left(\xi_{w}\right)_{\mathbb{P}}\right) \rightarrow \tilde{\Delta}_{*}\left(\mathcal{O}_{\left(X_{w}\right) \mathbb{P}}\right) \rightarrow \tilde{\Delta}_{*}\left(\mathcal{O}_{\left(\partial X_{w}\right) \mathbb{P}}\right) \rightarrow 0
$$

Tensor the sequence (16) with $\hat{p}^{*}\left(\mathcal{O}_{\mathbb{P}_{j}}\right)$ over $\mathcal{O}_{Y_{\mathbb{P}}}$, which preserves exactness by the proof of Lemma 4.9, and then tensor with $\gamma_{*} \mathcal{O}_{\left(X^{u} \times X^{v}\right)_{\mathbb{P}}}$ over $\mathcal{O}_{\bar{Y}_{\mathbb{P}}}$, which preserves exactness by Theorem 6.2 For closed subschemes $X$ and $Y$ of a scheme $Z$,

$$
\mathcal{O}_{X} \otimes_{\mathcal{O}_{Z}} \mathcal{O}_{Y}=\mathcal{O}_{X \cap Y}
$$

Thus,

$$
\gamma_{*} \mathcal{O}_{\left(X^{u} \times X^{v}\right)_{\mathbb{P}}} \otimes_{\mathcal{O}_{\bar{Y}}}\left(\hat{p}^{*}\left(\mathcal{O}_{\mathbb{P}_{j}}\right) \otimes_{\mathcal{O}_{Y_{\mathbb{P}}}} \tilde{\Delta}_{*}\left(\mathcal{O}_{\left(X_{w}\right)_{\mathbb{P}}}\right)\right)=\mathcal{O}_{\gamma\left(X^{u} \times X^{v}\right)_{\mathbb{P}} \cap \tilde{\Delta}\left(\left(X_{w}\right)_{j}\right)}=\mathcal{O}_{N_{\gamma}}
$$

and

$\gamma_{*} \mathcal{O}_{\left(X^{u} \times X^{v}\right)_{\mathbb{P}}} \otimes_{\mathcal{O}_{\bar{Y}_{\mathbb{P}}}}\left(\hat{p}^{*}\left(\mathcal{O}_{\mathbb{P}_{j}}\right) \otimes_{\mathcal{O}_{Y_{\mathbb{P}}}} \tilde{\Delta}_{*}\left(\mathcal{O}_{\left(\partial X_{w}\right)_{\mathbb{P}}}\right)\right)=\mathcal{O}_{\gamma\left(X^{u} \times X^{v}\right)_{\mathbb{P}} \cap \tilde{\Delta}\left(\left(\partial X_{w}\right)_{j}\right)}=\mathcal{O}_{M_{\gamma}^{2}}$.

Thus, (14) follows.

Similarly, (15) follows by tensoring the sequence (16) with $\hat{p}^{*}\left(\mathcal{O}_{\partial \mathbb{P}_{j}}\right)$ over $\mathcal{O}_{Y_{\mathbb{P}}}$, which preserves exactness by the proof of Lemma 4.9, and then tensoring with $\gamma_{*} \mathcal{O}_{\left(X^{u} \times X^{v}\right)_{\mathbb{P}}}$ over $\mathcal{O}_{\bar{Y}_{\mathbb{P}}}$, which preserves exactness by Theorem 6.2. This completes the proof.

By the previous lemma, Theorem 5.1 part b) is equivalent to the following theorem:

Theorem 9.2. For general $\gamma \in \bar{\Gamma}$ and any $u, v, w \in W, j \in[N]^{r}$ such that $c_{u, v}^{w}(j) \neq$ 0 we have

$$
H^{p}\left(N_{\gamma}, \mathcal{O}_{N_{\gamma}}\left(-M_{\gamma}\right)\right)=0
$$

for all $p \neq|j|+\ell(w)-\ell(u)-\ell(v)$.

Proof. First, the theorem is equivalent to the statement that for general $\gamma \in \bar{\Gamma}$,

$$
H^{p}\left(N_{\gamma}, \omega_{N_{\gamma}}\left(M_{\gamma}\right)\right)=0, \forall p>0 .
$$

To see this, observe that:

$$
\begin{aligned}
H^{p}\left(N_{\gamma}, \omega_{N_{\gamma}}\left(M_{\gamma}\right)\right) & =H^{p}\left(N_{\gamma}, \mathscr{H} \operatorname{om}_{\mathcal{O}_{N_{\gamma}}}\left(\mathcal{O}_{N_{\gamma}}\left(-M_{\gamma}\right), \omega_{N_{\gamma}}\right)\right) \\
& \stackrel{\varphi_{1}}{\simeq} \operatorname{Ext}_{N_{\gamma}}^{p}\left(\mathcal{O}_{N_{\gamma}}\left(-M_{\gamma}\right), \omega_{N_{\gamma}}\right) \\
& \stackrel{\varphi_{2}}{\simeq} H^{n-p}\left(N_{\gamma}, \mathcal{O}_{N_{\gamma}}\left(-M_{\gamma}\right)\right)^{*}
\end{aligned}
$$

where $n:=\operatorname{dim} N_{\gamma}=|j|+\ell(w)-\ell(u)-\ell(v)$, the isomorphism $\varphi_{1}$ follows by Corollary 7.11 and the local to global Ext spectral sequence [Go, Théorème 7.3.3, Chap. II], and the isomorphism $\varphi_{2}$ follows by Corollary [.10 and Serre duality [H, Chap. III, Theorem 7.6].

We now prove (17), which implies the theorem. By [S, Chapter I, $\S 6.3$, Theorem 1.25] and [H, Chap. III, Exercise 10.9], there is a non-empty open subset $\mathcal{U} \subset \bar{\Gamma}$ such that $\pi: \pi^{-1}(\mathcal{U}) \rightarrow \mathcal{U}$ is flat. (Observe that, by the proof of Corollary [7.10, $\pi$ is surjective.) We prove that $\omega_{\mathcal{Z}}(\partial \mathcal{Z})$ is flat over $\mathcal{U}$ :

To show this, let $A=\mathcal{O}_{\mathcal{U}}, B=\mathcal{O}_{\pi^{-1}(\mathcal{U})}$, and $M=\left.\omega_{\mathcal{Z}}(\partial \mathcal{Z})\right|_{\pi^{-1}(\mathcal{U})}$. By taking stalks, we immediately reduce to showing that for an embedding of local rings 
$A \subset B$ such that $A$ is regular and $B$ is flat over $A$, we have that $M$ is flat over $A$. Now, to prove this, let $\left\{x_{1}, \ldots, x_{d}\right\}$ be a minimal set of generators of the maximal ideal of $A$. Let $K_{\bullet}=K_{\bullet}\left(x_{1}, \ldots, x_{d}\right)$ be the Koszul complex of the $x_{i}$ 's over $A$. Then, recall that a finitely generated $B$-module $N$ is flat over $A$ iff $K_{\bullet} \otimes_{A} N$ is exact except at the extreme right, i.e., $H^{i}\left(K_{\bullet} \otimes_{A} N\right)=0$ for $i<d[\mathrm{E}$, Corollary 17.5 and Theorem 6.8]. Thus, by hypothesis, $K_{\bullet} \otimes_{A} B$ is exact except at the extreme right and hence the $x_{i}$ 's form a $B$-regular sequence [E, Theorem 17.6]. Now, since $\mathcal{O}_{\mathcal{Z}}$ and $\mathcal{O}_{\partial \mathcal{Z}}$ are $\mathrm{CM}$, we have that $\mathcal{O}_{\mathcal{Z}}(-\partial \mathcal{Z})$ is a $\mathrm{CM} \mathcal{O}_{\mathcal{Z}}$-module. Thus, by [I. Proposition 11.33], we have that $M$ is a CM $B$-module of dimension equal to $\operatorname{dim} B$. Therefore, by [I, Exercise 11.36], the $x_{i}$ 's form a regular sequence on the $B$-module $M$. Hence, $\left(K_{\bullet} \otimes_{A} B\right) \otimes_{B} M \simeq K_{\bullet} \otimes_{A} M$ is exact except at the extreme right [E, Corollary 17.5]. This proves that $M$ is flat over $A$, as desired.

Thus, by Corollary 8.8 and the semicontinuity theorem [Ke, Theorem 13.1] to prove (17), it is sufficient to show that for general $\gamma \in \bar{\Gamma}$, that

$$
\left.\omega_{\mathcal{Z}}(\partial \mathcal{Z})\right|_{\pi^{-1}(\gamma)} \simeq \omega_{N_{\gamma}}\left(M_{\gamma}\right) .
$$

To prove this, observe that since $\mathcal{U}$ is smooth and $\mathcal{Z}$ and $\partial \mathcal{Z}$ are $\mathrm{CM}$, and the assertion is local in $\mathcal{U}$, it suffices to observe (cf. [I. Corollary 11.35]) that for a non-zero function $\theta$ on $\mathcal{U}$, the sheaf

$$
\mathcal{S} / \theta \cdot \mathcal{S}=\mathscr{H} \text { om }_{\mathcal{O}_{\mathcal{Z}_{\theta}}}\left(\mathcal{O}_{\mathcal{Z}}(-\partial \mathcal{Z}) / \theta \cdot \mathcal{O}_{\mathcal{Z}}(-\partial \mathcal{Z}), \omega_{\mathcal{Z}_{\theta}}\right)
$$

where $\mathcal{Z}_{\theta}$ denotes the zero scheme of $\theta$ in $\mathcal{Z}$ and the sheaf

$$
\mathcal{S}:=\mathscr{H}_{o m_{\mathcal{Z}}}\left(\mathcal{O}_{\mathcal{Z}}(-\partial \mathcal{Z}), \omega_{\mathcal{Z}}\right) \text {. }
$$

Choosing $\theta$ to be in a local coordinate system and using induction and the above result, the desired conclusion (18) is obtained.

\section{ACKNOWLEDGMENTS}

The authors would like to thank D. Anderson for providing some clarifications and additional details regarding their paper [AGM] and N. Mohan Kumar for several helpful correspondences. This work was partially supported by NSF grant number DMS-1501094.

\section{REFERENCES}

[AGM] D. Anderson, S. Griffeth, and E. Miller, Positivity and Kleiman transversality in equivariant K-theory of homogeneous spaces, J. Eur. Math. Soc. (JEMS) 13 (2011), no. 1, 57-84, DOI 10.4171/JEMS/244. MR2735076

[CG] N. Chriss and V. Ginzburg, Representation theory and complex geometry, Birkhäuser Boston, Inc., Boston, MA, 1997. MR 1433132

[D] O. Debarre, Higher-dimensional algebraic geometry, Universitext, Springer-Verlag, New York, 2001. MR1841091

[E] D. Eisenbud, Commutative algebra, With a view toward algebraic geometry, Graduate Texts in Mathematics, vol. 150, Springer-Verlag, New York, 1995. MR1322960

[El] R. Elkik, Singularités rationnelles et déformations (French), Invent. Math. 47 (1978), no. 2, 139-147, DOI 10.1007/BF01578068. MR501926

[EV] H. Esnault and E. Viehweg, Lectures on vanishing theorems, DMV Seminar, vol. 20, Birkhäuser Verlag, Basel, 1992. MR1193913

[FP] W. Fulton and P. Pragacz, Schubert varieties and degeneracy loci, Lecture Notes in Mathematics, vol. 1689, Springer-Verlag, Berlin, 1998. Appendix J by the authors in collaboration with I. Ciocan-Fontanine. MR 1639468

[Go] R. Godement, Topologie algébrique et théorie des faisceaux (French), Actualités Sci. Ind. No. 1252. Publ. Math. Univ. Strasbourg. No. 13, Hermann, Paris, 1958. MR0102797 
[GK] W. Graham and S. Kumar, On positivity in T-equivariant K-theory of flag varieties, Int. Math. Res. Not. IMRN, posted on 2008, Art. ID rnn 093, 43, DOI 10.1093/imrn/rnn093. MR 2439542

[GR] S. Griffeth and A. Ram, Affine Hecke algebras and the Schubert calculus, European J. Combin. 25 (2004), no. 8, 1263-1283, DOI 10.1016/j.ejc.2003.10.012. MR 2095481

$[\mathrm{H}] \quad$ R. Hartshorne, Algebraic geometry, Springer-Verlag, New York-Heidelberg, 1977. Graduate Texts in Mathematics, No. 52. MR0463157

[I] S. B. Iyengar, G. J. Leuschke, A. Leykin, C. Miller, E. Miller, A. K. Singh, and U. Walther, Twenty-four hours of local cohomology, Graduate Studies in Mathematics, vol. 87, American Mathematical Society, Providence, RI, 2007. MR2355715

[K] M. Kashiwara, The flag manifold of Kac-Moody Lie algebra, Algebraic analysis, geometry, and number theory (Baltimore, MD, 1988), Johns Hopkins Univ. Press, Baltimore, MD, 1989, pp. 161-190. MR1463702

[KS] M. Kashiwara and M. Shimozono, Equivariant K-theory of affine flag manifolds and affine Grothendieck polynomials, Duke Math. J. 148 (2009), no. 3, 501-538, DOI 10.1215/00127094-2009-032. MR2527324

[Ke] G. Kempf, The Grothendieck-Cousin complex of an induced representation, Adv. in Math. 29 (1978), no. 3, 310-396, DOI 10.1016/0001-8708(78)90021-X. MR509802

[KM] J. Kollár and S. Mori, Birational geometry of algebraic varieties, Cambridge Tracts in Mathematics, vol. 134, Cambridge University Press, Cambridge, 1998. With the collaboration of C. H. Clemens and A. Corti; Translated from the 1998 Japanese original. MR 1658959

[Ku1] S. Kumar, Positivity in T-equivariant K-theory of flag varieties associated to Kac-Moody groups. Preprint (2012). (To appear in J. Euro. Math. Soc.)

[Ku2] S. Kumar, Kac-Moody groups, their flag varieties and representation theory, Progress in Mathematics, vol. 204, Birkhäuser Boston, Inc., Boston, MA, 2002. MR.1923198

[KuS] S. Kumar and K. Schwede, Richardson varieties have Kawamata log terminal singularities, Int. Math. Res. Not. IMRN 3 (2014), 842-864. MR3163569

[LSS] T. Lam, A. Schilling, and M. Shimozono, K-theory Schubert calculus of the affine Grassmannian, Compos. Math. 146 (2010), no. 4, 811-852, DOI 10.1112/S0010437X09004539. MR 2660675

[S] I. R. Shafarevich, Basic algebraic geometry. 1, Varieties in projective space, third ed., Springer-Verlag, Berlin, 2013. Translated from the 1988 Russian edition and with notes by Miles Reid.

[Se] J. P. Serre. Espaces fibrés algébriques, Séminaire C. Chevalley, E.N.S., 1958.

[Stacks] The Stacks Project, http://stacks.math.columbia.edu/ Accessed June 15, 2016.

Department of Mathematics, University of North Carolina, Chapel Hill, North CarOLINA 27599-3250

E-mail address: seth.baldwin@unc.edu

Department of Mathematics, University of North Carolina, Chapel Hill, North CarOLINA 27599-3250

E-mail address: shrawan@email.unc.edu 